\title{
Cross-ambiguity function domain multipath channel parameter estimation
}

\author{
Mehmet Burak Guldogan ${ }^{\mathrm{a}, *}$, Orhan Arikan ${ }^{\mathrm{b}}$ \\ a Department of Electrical Engineering, Linköping University, Linköping 58183, Sweden \\ ${ }^{\mathrm{b}}$ Department of Electrical and Electronics Engineering, Bilkent University, Bilkent, Ankara TR-06800, Turkey
}

\section{A R T I C L E I N F O}

\section{Article history:}

Available online 23 November 2011

\section{Keywords:}

Direction-of-arrival (DOA)

Multipath channels

Channel estimation

Cross-ambiguity function (CAF)

Space-alternating generalized

expectation-maximization (SAGE) algorithm

\begin{abstract}
A B S T R A C T
A new array signal processing technique is proposed to estimate the direction-of-arrivals (DOAs), time delays, Doppler shifts and amplitudes of a known waveform impinging on an array of antennas from several distinct paths. The proposed technique detects the presence of multipath components by integrating cross-ambiguity functions (CAF) of array outputs, hence, it is called as the cross-ambiguity function direction finding (CAF-DF). The performance of the CAF-DF technique is compared with the space-alternating generalized expectation-maximization (SAGE) and the multiple signal classification (MUSIC) techniques as well as the Cramér-Rao lower bound. The CAF-DF technique is found to be superior in terms of root-mean-squared-error (rMSE) to the SAGE and MUSIC techniques.
\end{abstract}

(C) 2011 Elsevier Inc. All rights reserved.

\section{Introduction}

The next generation mobile radio communication systems are faced with the ever increasing demand for higher communication rates. In order to meet this demand, the communication systems should obtain an accurate model for the communication channel [1]. For fuller utilization of multipath communication channels, communication systems utilize antenna arrays and sophisticated signal processing techniques to produce estimates for multipath channel parameters including direction-of-arrivals (DOA), time delays, Doppler shifts and amplitudes. There are a multitude of array signal processing techniques proposed for reliable and accurate estimation for these channel parameters. Multipath channel parameter estimation techniques can be grouped into three categories [2]: spectral-based estimation, parametric subspace-based estimation and deterministic parametric estimation. Conventional beamformer, Capon's beamformer [3] and MUSIC [4] can be stated within the first category. In contrast to beamforming techniques, MUSIC algorithm provides statistically consistent estimates and became a highly popular algorithm [5-9]. The signal subspace fitting (SSF) [10], weighted subspace fitting (WSF) [11], estimation of signal parameter estimation via rotational invariance techniques (ESPRIT) [12] and unitary ESPRIT [13] are computationally efficient techniques and belong to the second category. In the last category, maximum likelihood (ML) techniques should be stated $[2,14]$. Compared to all other estimation methods, ML has the superior asymptotic performance. The major issue of the ML technique is its high computational complexity associated with the direct maximization of likelihood function. Various indirect maximization

\footnotetext{
* Corresponding author. Fax: +46(0)13 139282.

E-mail address: bguldogan@isy.liu.se (M.B. Guldogan).
}

techniques, such as, data-supported grid search [15], alternating projection method [16], and simulated annealing algorithms [17] are proposed to obtain the ML estimates more efficiently. However, these approaches cannot guarantee global convergence in general.

Alternatively, a very popular method to facilitate simple implementation of likelihood function is the expectation maximization (EM) algorithm formulated by Dempster et al. [18]. The maximization of the original likelihood function can be replaced by simpler maximizations in lower dimensional parameter spaces. To further improve the performance of the EM algorithm various techniques have been developed [19-21]. The SAGE algorithm, which was developed by Fessler and Hero [22], is a very powerful technique to further improve the EM algorithm. It sequentially estimates the channel parameters, whereas the EM simultaneously updates all parameters. Main advantage of the SAGE algorithm compared to the EM algorithm is its faster convergence resulting in a correspondingly lower complexity. The SAGE algorithm has been applied for joint channel parameter estimation and some of these efforts are reported in [19,23-29].

In wideband communication channels with peak power limitations, typically coded waveforms with the time-bandwidth products significantly larger than one are employed. In these systems, pulse compression of the receiver is a necessity to provide optimal extraction of the transmitted information. In delay only channels with bandlimited white noise, pulse compression can be achieved by a simple matched filter that implements correlation of the incoming signal with the transmitted waveform. However, in the presence of Doppler shifts, a single matched filter cannot provide the optimal performance, rather a bank of matched filters each matched to a specific Doppler shift should be employed [30], providing individual Doppler slices of the CAF between the transmitted and received signals. Therefore, it is of both theoretical 
and practical interest to develop array signal processing techniques where CAF is an integrated component of the processing chain [31]. Here we present details of the proposed technique and comparison results of it with commonly used the SAGE and the MUSIC techniques, where two criterions used to quantitatively measure the performance. The first criterion is based on the accuracy of the obtained channel parameters. The second criterion is based on the overall performance of a communication receiver which utilizes the estimated channel parameters to form its decision on the transmitted information bit.

The paper is organized as follows. The parametric channel model is outlined in Section 2. Details of the CAF-DF algorithm are presented in Section 3. Performance results of each technique are presented in Section 4. Details of the MUSIC-based and the SAGE algorithms are given in Appendices A and B, respectively. The CRLB derivations are provided in Appendix $C$.

\section{Parametric channel model}

In this section, we describe a commonly used parametric model for multipath channels. For this purpose, we will consider transmitted signals that can be written as a train of modulated pulses:

$s(t)=\sum_{k=1}^{q} b_{k} p(t-(k-1) T)$,

where $p(t)$ is the modulated pulse with time-bandwidth product is larger than 1 , and $b_{k}$ are \pm 1 . In a typical communication system $\left\{b_{k}: 1 \leqslant k \leqslant q\right\}$ are set differently for different transmitters sharing the same channel. In the following, we will assume that there is only one transmitter in the channel with $b_{k}=1,1 \leqslant k \leqslant q$. In this way, we will be able to provide our main results with significantly less notational complexity. In Fig. 1, delayed, Doppler shifted and attenuated copies of the transmitted signal from a transmitter (TX) impinge on $M$ element receiver antenna array ( $\mathrm{RX}$ ) from different paths. Under the narrowband assumption which is valid when the reciprocal of the bandwidth is much bigger than the propagation of the waveform across the array, passband output signal of the $m$ th receiving antenna can be modeled as:

$$
\begin{aligned}
x_{m}(t)= & \sum_{i=1}^{d} \sum_{k=1}^{q} \zeta_{i} p\left(t-\tau_{m, i}-(k-1) T\right) \\
& \times e^{j 2 \pi v_{m, i}\left(t-\xi_{m, i}\left(\theta_{i}, \phi_{i}\right)\right)} e^{j 2 \pi v_{c}\left(t-\xi_{m, i}\left(\theta_{i}, \phi_{i}\right)\right)}+n_{m}(t),
\end{aligned}
$$

where the parameters are defined as follows:

- $d$ : number of different multipath signals,

- $q$ : number of coded pulses in the transmitted signal,

- $\zeta_{i}$ : complex scaling factor of the $i$ th path containing all the attenuation and phase terms,

- $p$ : coded pulse signal,

- $\tau_{m, i}$ : time delay of the $i$ th path for the $m$ th antenna,

- $T$ : pulse repetition interval in seconds,

- $v_{m, i}$ : Doppler shift of the $i$ th path for the $m$ th antenna,

- $\theta_{i}$ : azimuth angle in degrees,

- $\phi_{i}$ : elevation angle in degrees,

- $\xi_{m, i}$ : relative phase of the $m$ th antenna due to the $i$ th impinging signal with respect to the origin of the antenna array,

- $v_{c}$ : carrier frequency,

- $n_{m}$ : complex additive noise, which is assumed to be spatially and temporally white Gaussian distributed with covariance $\sigma^{2}$.

DOA $\left(\theta_{i}, \phi_{i}\right)$ and the speed of an impinging electromagnetic wave on an antenna array can be defined by the vector, $\boldsymbol{\alpha}=$ $\mathrm{c}^{-1}\left[\cos \left(\theta_{i}\right) \cos \left(\phi_{i}\right), \sin \left(\theta_{i}\right) \cos \left(\phi_{i}\right), \sin \left(\phi_{i}\right)\right]$, called as the slowness

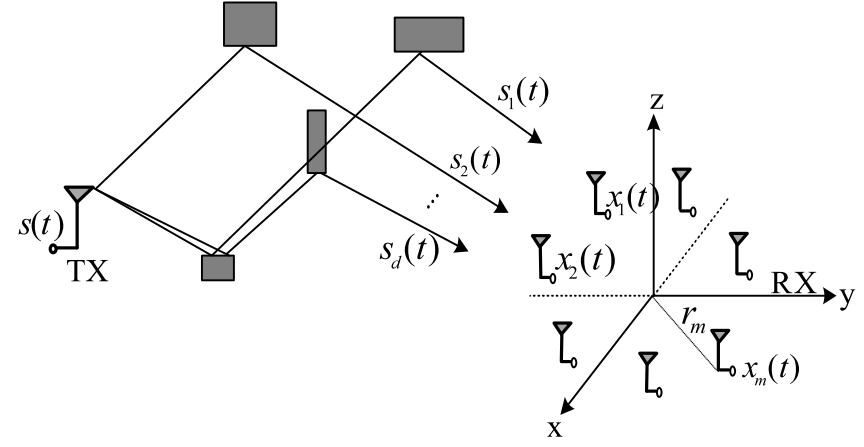

Fig. 1. An illustration of the receiver antenna array (RX) intercepting multipath signals from the transmitter (TX).

vector based on the reference coordinate system in Fig. 1. Position of the each antenna in the array is represented with the vector $\mathbf{r}_{m}=r_{m}\left[\cos \left(\theta_{m}^{a}\right), \sin \left(\theta_{m}^{a}\right), 0\right]^{T}$. Here, $r_{m}$ is the distance of the $m$ th antenna to the array origin, $\left(\theta_{m}^{a}\right)$ is the angle on the $x$-y-axis from $x$-axis to the antenna and superscript $a$ denotes antenna. Assuming that the relative orientations of the antennas are known, relative phase of the $m$ th antenna due to the $i$ th impinging signal with respect to the origin of the antenna array $\xi_{m, i}$ can be written as

$$
\begin{aligned}
\xi_{m, i}\left(\theta_{i}, \phi_{i}\right)= & \boldsymbol{\alpha}_{i} \mathbf{r}_{m} \\
= & \frac{r_{m}}{c}\left(\cos \left(\theta_{i}\right) \cos \left(\phi_{i}\right) \cos \left(\theta_{m}^{a}\right)\right. \\
& \left.+\sin \left(\theta_{i}\right) \cos \left(\phi_{i}\right) \sin \left(\theta_{m}^{a}\right)\right),
\end{aligned}
$$

where $\left(\theta_{i}, \phi_{i}\right)$ is the DOA for the $i$ th path and $c$ is the propagation velocity in the channel. The received signal model given in (2) can be further simplified under mild assumptions. For narrowband signals, the time delay appears as a pure phase delay of the reference signal. Moreover, this phase delay depends only on the spacing between the array elements and the DOA of the plane wave. Therefore, the same delay can be used across the receiving antennas which is the delay, $\tau_{i}$, between the transmitter and the array origin. Since the receiving antennas are closely spaced, the Doppler shifts across the receiving antennas corresponding to an impinging signal can be taken the same $v_{m, i}=v_{i}$. Furthermore, for typical Doppler spreads encountered in multipath wireless communications $e^{-j 2 \pi v_{m, i} \xi_{m, i}\left(\theta_{i}, \phi_{i}\right)}$ can be safely assumed to be one [32, 33]. Hence, under these conditions, received baseband signals at each antenna can be simplified to:

$$
\begin{aligned}
x_{m}(t)= & \sum_{i=1}^{d} \sum_{k=1}^{q} \zeta_{i} p\left(t-\tau_{i}-(k-1) T\right) \\
& \times e^{j 2 \pi v_{i} t} e^{-j 2 \pi v_{c} \xi_{m, i}\left(\theta_{i}, \phi_{i}\right)}+n_{m}(t) .
\end{aligned}
$$

Then by using (1), array output can also be expressed as in the following vector-matrix form:

$$
\begin{aligned}
\mathbf{x}(t) & =\sum_{i=1}^{d} \mathbf{a}\left(\theta_{i}, \phi_{i}\right) \zeta_{i} s\left(t-\tau_{i}\right) e^{j 2 \pi v_{i} t}+\mathbf{n}(t) \\
& =\mathbf{A s}(t)+\mathbf{n}(t),
\end{aligned}
$$

where $\mathbf{A}=\left[\mathbf{a}\left(\theta_{1}, \phi_{1}\right), \ldots, \mathbf{a}\left(\theta_{d}, \phi_{d}\right)\right]_{M \times d}$ is the steering matrix, $\mathbf{a}\left(\theta_{i}, \phi_{i}\right)=\left[a_{1}\left(\theta_{i}, \phi_{i}\right), \ldots, a_{M}\left(\theta_{i}, \phi_{i}\right)\right]^{T}$ is the spatial signature of the $i$ th arrival, $\mathbf{n}(t)=\left[n_{1}(t), \ldots, n_{M}(t)\right]^{T}$ is the noise vector, $\mathbf{s}(t)=$ $\left[s_{1}(t), \ldots, s_{d}(t)\right]^{T}$ is the vector of delayed and Doppler shifted signals: $s_{i}(t)=\zeta_{i} s\left(t-\tau_{i}\right) e^{j 2 \pi v_{i} t}$. Assuming $N$ snapshots are taken, a more compact matrix representation can be written as

$\mathbf{X}=\mathbf{A S}+\mathbf{N}$, 
where $\mathbf{X}$ and $\mathbf{N}$ are $M \times N$ matrices with each snapshot forming a column:

$\mathbf{X}=\left[\mathbf{x}\left(t_{1}\right), \ldots, \mathbf{x}\left(t_{N}\right)\right]$,

$\mathbf{N}=\left[\mathbf{n}\left(t_{1}\right), \ldots, \mathbf{n}\left(t_{N}\right)\right]$,

and $\mathbf{S}$ is the $d \times N$ signal matrix, $\mathbf{S}=\left[\mathbf{s}\left(t_{1}\right), \ldots, \mathbf{s}\left(t_{N}\right)\right]$. Moreover, spatial covariance matrix of the array output can be given as:

$$
\begin{aligned}
\mathbf{R}^{S} & =E\left\{\mathbf{X} \mathbf{X}^{H}\right\} \\
& =\mathbf{A} E\left\{\mathbf{S S}^{H}\right\} \mathbf{A}^{H}+E\left\{\mathbf{N N}^{H}\right\},
\end{aligned}
$$

where $E\{$.$\} represents the statistical averaging operation. Covari-$ ance matrix of the received signal is usually unavailable. Therefore, usually sample covariance matrix is used and can be estimated as

$\hat{\mathbf{R}}^{s}=\frac{1}{N} \mathbf{X X}^{H}$.

Our primary aim is to accurately estimate the time delays $\tau_{1}$, $\ldots, \tau_{d}$, Doppler shifts $v_{1}, \ldots, v_{d}$ and DOAs $\left(\theta_{1}, \phi_{1}\right), \ldots,\left(\theta_{d}, \phi_{d}\right)$ of the incoming signals from the $N$ snapshots of the array $\mathbf{x}\left(t_{1}\right), \ldots, \mathbf{x}\left(t_{N}\right)$. To solve this problem we assume that the following conditions hold:

(A1) The number of paths $d$ is known.

(A2) The array manifold $\mathbf{A}(\theta, \phi)$ is known.

Note that the first assumption is the most restrictive one. Although we will not go into detail here, there are excellent techniques to determine the number of paths [34-37]. An important performance criterion in multipath channel parameter estimation is the effect of the estimated channel parameters to the performance of the communication receiver system where the estimated channel parameters can be used to form the following decision metric:

$\hat{\rho}=\int_{0}^{q T} s^{H}(t)\left(\sum_{m=1}^{M} \sum_{i=1}^{d} \hat{\zeta}_{i}^{H} x_{m}\left(t+\hat{\tau}_{i}\right) e^{-j 2 \pi \hat{v}_{i} t} a_{m}^{H}\left(\hat{\theta}_{i}, \hat{\phi}_{i}\right)\right) d t$

where $(\hat{.})$ represents the estimated value of the parameter inside the parentheses and $(\cdot)^{H}$ is the conjugate transpose operator. This decision signal is very similar to the decision signal generated by a rake receiver [38]. Here we employed a raking strategy in both delay and Doppler as well as between various DOAs of the multipath components. Eq. (12) can also be written as:

$$
\begin{aligned}
\hat{\rho}= & \rho_{s}+\rho_{n} \\
= & \int_{0}^{q T} s^{H}(t)\left(\sum_{m=1}^{M} \sum_{i=1}^{d} \sum_{l=1}^{d} \hat{\zeta}_{i}^{H} \zeta_{l} s\left(t-\tau_{l}+\hat{\tau}_{i}\right) e^{j 2 \pi\left(v_{l}-\hat{v}_{i}\right) t}\right. \\
& \left.\times a_{m}^{H}\left(\hat{\theta}_{i}, \hat{\phi}_{i}\right) a_{m}\left(\theta_{l}, \phi_{l}\right)\right) d t \\
& +\int_{0}^{q T} s^{H}(t)\left(\sum_{m=1}^{M} \sum_{i=1}^{d} \hat{\zeta}_{i}^{H} n_{m}\left(t+\hat{\tau}_{i}\right) e^{-j 2 \pi \hat{v}_{i} t} a_{m}^{H}\left(\hat{\theta}_{i}, \hat{\phi}_{i}\right)\right) d t \\
= & \rho_{s}+\sum_{m=1}^{M} \sum_{i=1}^{d} \hat{\zeta}_{i}^{H} a_{m}^{H}\left(\hat{\theta}_{i}, \hat{\phi}_{i}\right) \int_{0}^{q T} s^{H}(t) n_{m}\left(t+\hat{\tau}_{i}\right) e^{-j 2 \pi \hat{v}_{i} t} d t \\
= & \rho_{s}+\sum_{m=1}^{M} \sum_{i=1}^{d} \hat{\zeta}_{i}^{H} a_{m}^{H}\left(\hat{\theta}_{i}, \hat{\phi}_{i}\right) h_{m, i},
\end{aligned}
$$

where $h_{m, i}$ are i.i.d. random variables for all $m$ and $i$, with $E\left\{h_{m, i}\right\}=0, E\left\{\left|h_{m, i}\right|^{2}\right\}=\sigma^{2} \int_{0}^{q T}|s(t)|^{2} d t=\sigma^{2} E_{s}$. Variance of the noise in Eq. (13) is:

$$
\begin{aligned}
\operatorname{Var}\left\{\rho_{n}\right\}= & E\left\{\left(\sum_{m=1}^{M} \sum_{i=1}^{d} \hat{\zeta}_{i}^{H} a_{m}^{H}\left(\hat{\theta}_{i}, \hat{\phi}_{i}\right) h_{m, i}\right)\right. \\
& \left.\times\left(\sum_{m^{\prime}=1}^{M} \sum_{i^{\prime}=1}^{d} \hat{\zeta}_{i^{\prime}} a_{m^{\prime}}\left(\hat{\theta}_{i^{\prime}}, \hat{\phi}_{i^{\prime}}\right) h_{m^{\prime}, i^{\prime}}^{H}\right)\right\} \\
= & M \sigma^{2} E_{s} \sum_{i=1}^{d}\left|\zeta_{i}\right|^{2} .
\end{aligned}
$$

The estimated or decision SNR given below serves as a performance criterion between alternative techniques:

$\widehat{\mathrm{SNR}}=\frac{|\hat{\rho}|^{2}}{\operatorname{Var}\left\{\rho_{n}\right\}}=\frac{|\hat{\rho}|^{2}}{E_{s} M \sigma^{2} \sum_{i=1}^{d}\left|\hat{\zeta}_{i}\right|^{2}}$

where $E_{s}$ is the transmitted signal energy.

\section{The cross-ambiguity function domain direction finding technique}

The cross-ambiguity function domain direction finding (CAF-DF) is an iterative technique, where at each iteration, parameters of a single path are estimated [39]. In the rest of this section, we present the steps of the technique in detail. The received signals are often modeled as delayed, Doppler shifted and scaled versions of the transmitted signal. As it is used in radar signal processing, the CAF can be used in order to estimate the time delay of a Doppler shifted signal for the received signal $x_{m}(t)$ and the transmitted signal $s(t)[30,40]$. In terms of SNR, matched filtering is the optimum solution for detection. When the Doppler shift is not known, performance of the receiver, that makes use of a matched filter matched to the transmitted signal, may significantly degrade. The ambiguity function characterizes the output of a matched filter when the input signal is delayed and Doppler shifted. In the case of one multipath component, CAF calculation is the optimal solution for detection. If there exist two or more multipath components separated enough in delay-Doppler domain, the CAF surface offers a very useful detection surface by using properly chosen waveforms for the application of interest $[30,41]$. Although there exist several different representations, we prefer symmetrical version of the CAF:

$\chi_{x_{m}, s}(\tau, v)=\int_{-\infty}^{\infty} x_{m}(t+\tau / 2) s^{H}(t-\tau / 2) e^{-j 2 \pi v t} d t$.

Starting point of the CAF-DF technique is to estimate the DOA information which is captured in $e^{-j 2 \pi v_{c} \xi_{m, i}\left(\theta_{i}, \phi_{i}\right)}$ of (4). This information will be gathered by collecting $M$ complex values from calculated CAFs on each antenna of the array. For this purpose, CAF with the known transmitted waveform is computed on each antenna. When the phase of each impinging signal on the array is unknown, to detect the delay and Doppler coordinates of a multipath component (highest peak point on the CAF surface), absolute value of each CAF calculated at the output of each antenna is incoherently integrated as:

$\chi_{\text {incoh }}(\tau, v)=\frac{1}{M} \sum_{m=1}^{M}\left|\chi_{x_{m}, s}(\tau, v)\right|$.

Coordinates of the peak of the $\chi_{\text {incoh }}(\tau, \nu)$ provide us initial delay-Doppler estimates of the path in consideration. These initial estimates are updated in the end of each iteration based on 


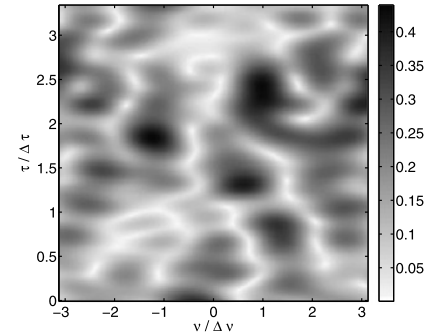

(a)

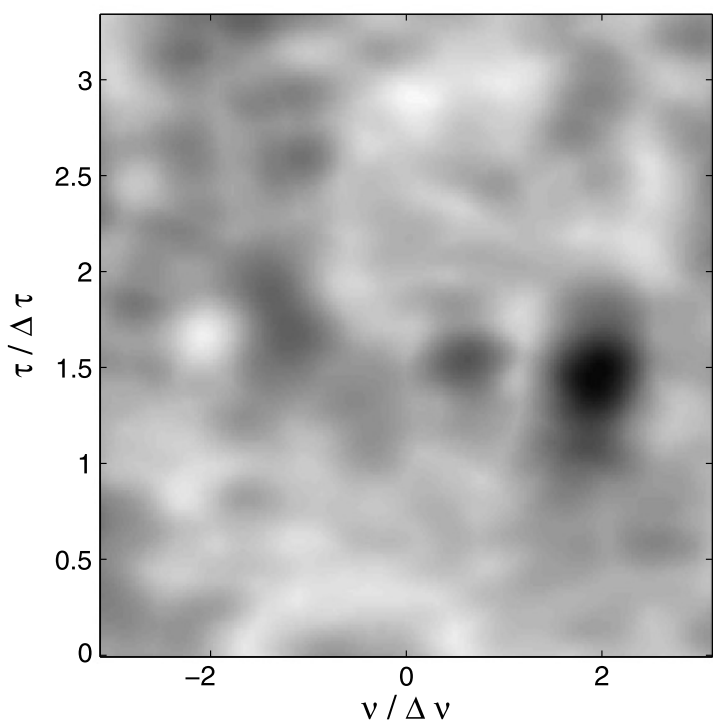

(e)

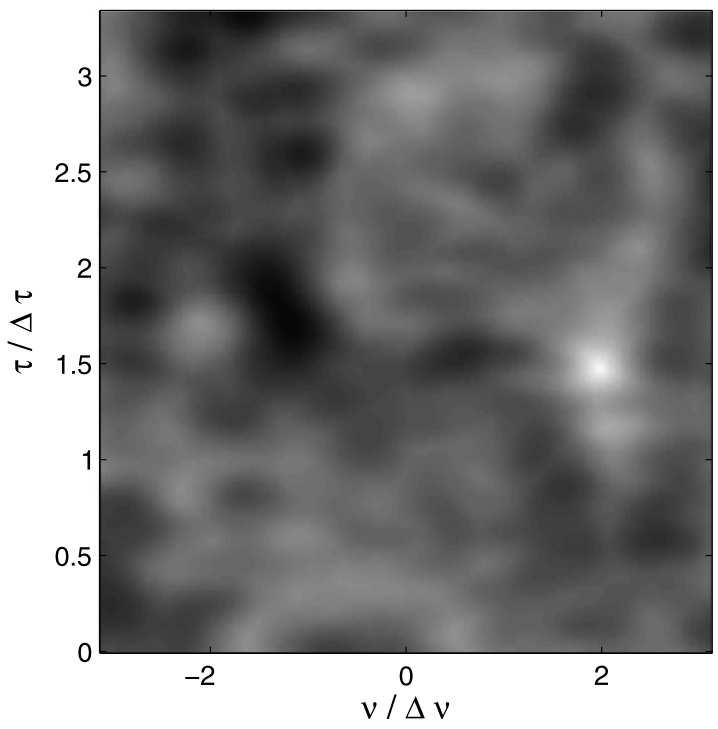

(g)

(b)
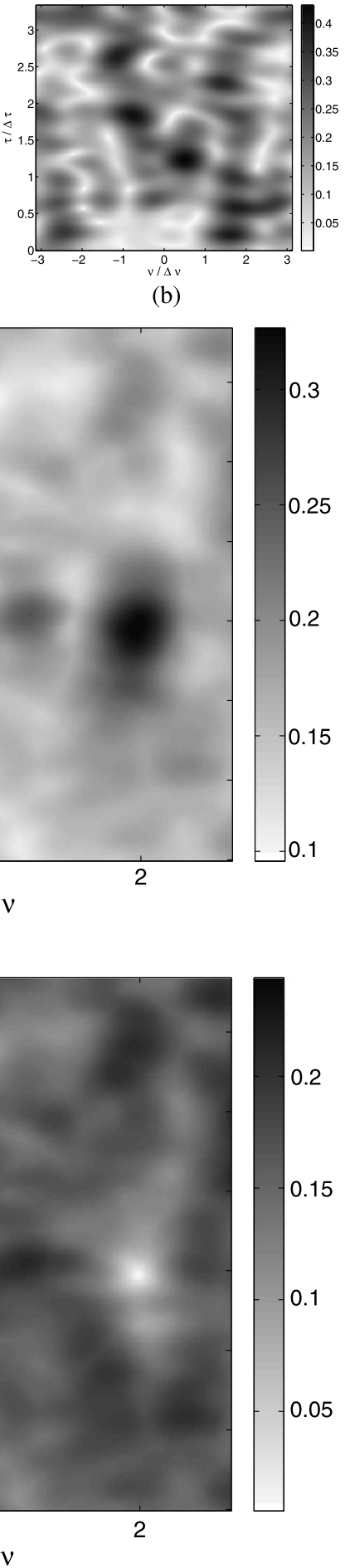

0.15

0.1

0.05

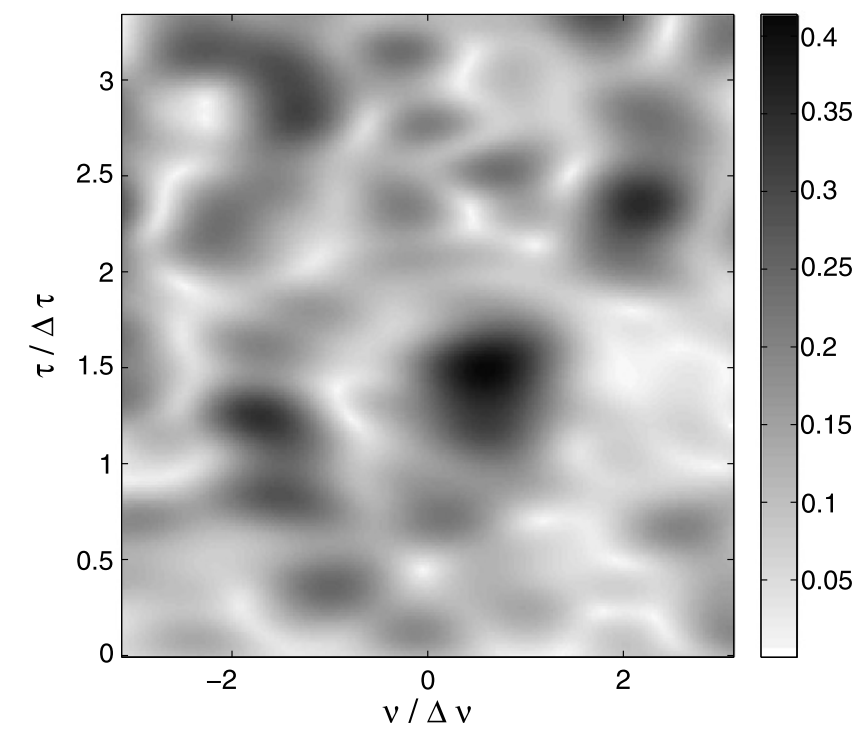

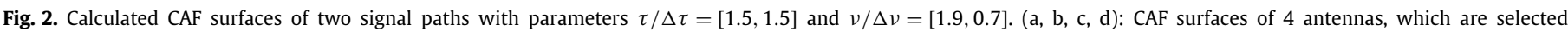

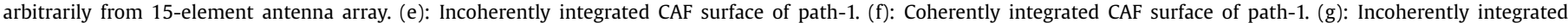
CAF surface of path-2. (h): Coherently integrated CAF surface of path-2.

a coherent integration process. Since antennas in the array are closely spaced, peak location of each CAF will be nearly the same for each antenna. This means that, after incoherent integration, desired highest peak power on the incoherently integrated CAF surface is not changed notably but noise power is reduced all around the highest peak. In another words, compared to detecting highest peak on a CAF surface, same probability of detection can be obtained with less SNR if detection of the highest point is performed (h)

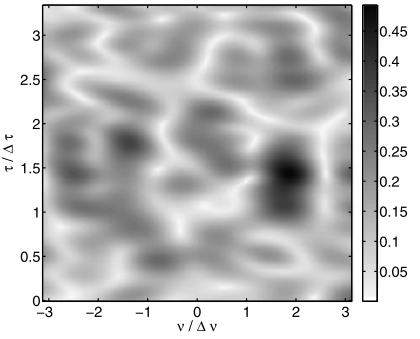

(c)

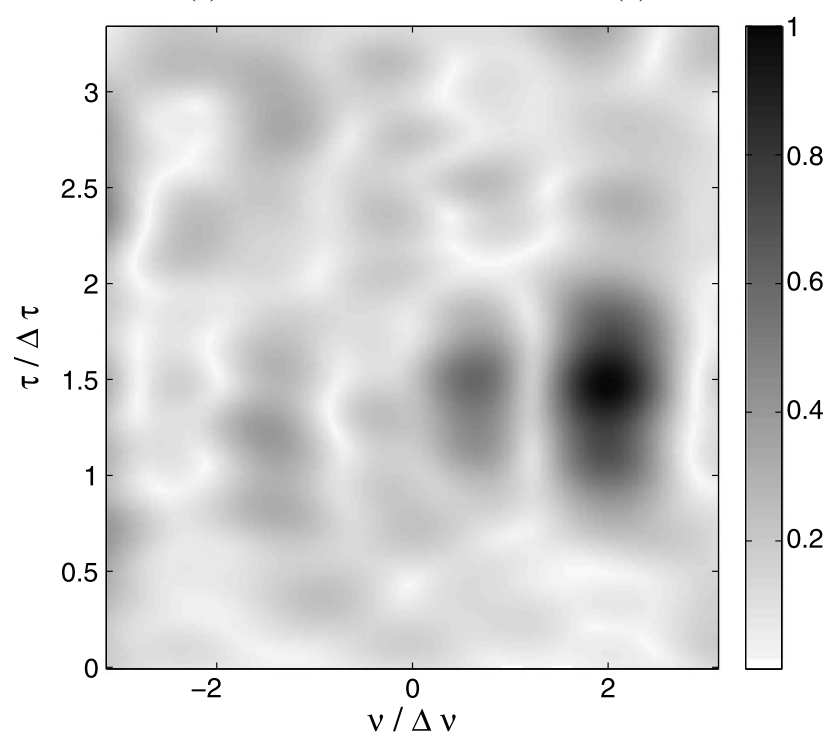

(f)

on the incoherently integrated CAF surface. Detection performance of the initial delay and Doppler coordinates $\left(\hat{\tau}_{i}^{\prime}, \hat{v}_{i}^{\prime}\right)$ of the highest peak that exceeds a predefined detection threshold is improved by this way. This practical approach is widely used in radar signal processing and there are excellent references presenting details of the procedure $[30,41,42]$. The incoherent integration-based peak detection procedure is illustrated in Fig. 2, by using a 15-element circular antenna array output. Note that the resolution of delay 
and Doppler in the CAF domain are $\Delta \tau=1 / \mathrm{BW}$ and $\Delta v=1 / T_{\text {coh }}$, respectively. Here, BW corresponds to the bandwidth and $T_{\text {coh }}$ is the duration of $s(t)$. One important principle that should be mentioned at this point is the uncertainty principle [30]. Briefly, it says that, if one narrow the peak on AF surface to increase the estimator's local accuracy, the volume removed will reappear somewhere away from the peak and will decrease the estimator's global accuracy. This behavior of the AF indicates that there have to be trade-offs made among the resolution, accuracy, and ambiguity. Therefore, type of the waveform determines the accuracy in resolving multipath components and should be chosen based on the interested problem. Maybe the most famous family of phase codes are the Barker codes [30]. In this paper, as a transmitted signal, $s(t)$, Barker-13 phase coded pulse train is used, which provides good delay and Doppler resolution for the interested area on the AF surface. Barker-13 phase coded waveforms yield a peak-topeak sidelobe ratio of 13. Fig. 2(a)-(d) are the sample CAF surfaces calculated for four antennas and Fig. 2(e), (g) are the incoherent integration of fifteen CAF surfaces for path-1 and path-2, respectively. It is seen from the resultant normalized CAF that the noise level is suppressed relative to the peak when compared to the individual CAFs. In short, peak detection on the incoherently integrated CAF surface provided more accurate initial delay-Doppler estimates $\left(\hat{\tau}_{i}^{\prime}, \hat{v}_{i}^{\prime}\right)$ of the $i$ th multipath component than an individually calculated CAF on one antenna, yielding more accurate DOA estimation.

As we have pointed out previously, receiver performance is severely degraded if Doppler effect is not an integral part of the processing chain. Therefore, joint delay-Doppler estimation is required to have optimal detection and accurate DOA estimation. Especially, at low SNR level, joint delay-Doppler processing using CAF significantly improves the success in separating overlapped (closely spaced) multipath components. By this way, accuracy in DOA estimation is significantly increased. In order to motivate the usage of incoherently integrated CAF processing, consider that the initial delay and Doppler parameters are estimated using separate optimizations:

$$
\begin{aligned}
& \hat{\tau}_{i}=\arg \max _{\tau} \sum_{m=1}^{M}\left|\int_{-\infty}^{+\infty} s^{H}(t-\tau) x_{m}(t) d t\right|, \\
& \hat{v}_{i}=\arg \max _{v} \sum_{m=1}^{M}\left|\int_{-\infty}^{+\infty} s^{H}\left(t-\hat{\tau}_{i}\right) e^{-j 2 \pi v t} x_{m}(t) d t\right| .
\end{aligned}
$$

Delay estimates in (18) are not accurate, due to not considering the Doppler effect. Moreover, delay estimation error is propagated to Doppler estimates in (19). Clearly, DOA estimation will include this propagated estimation error. However, in the CAF-DF technique, once the largest peak exceeding the detection threshold is identified, the following vector $\mathbf{P}_{\mathbf{i}}$ is formed from the complex values of individual CAF surfaces at the detected peak location $\left(\hat{\tau}_{i}^{\prime}, \hat{v}_{i}^{\prime}\right)$.

$$
\mathbf{P}_{\mathbf{i}}=\left[\boldsymbol{\chi}_{x_{1}, s}\left(\hat{\tau}_{i}^{\prime}, \hat{v}_{i}^{\prime}\right), \ldots, \chi_{x_{m}, s}\left(\hat{\tau}_{i}^{\prime}, \hat{v}_{i}^{\prime}\right), \ldots, \chi_{x_{M}, s}\left(\hat{\tau}_{i}^{\prime}, \hat{v}_{i}^{\prime}\right)\right]^{T}
$$

where

$$
\begin{aligned}
\chi_{x_{m}, s}\left(\hat{\tau}_{i}^{\prime}, \hat{v}_{i}^{\prime}\right) \\
=\int_{-\infty}^{+\infty} x_{m}\left(t+\hat{\tau}_{i}^{\prime} / 2\right) s^{H}\left(t-\hat{\tau}_{i}^{\prime} / 2\right) e^{-j 2 \pi \hat{v}_{i}^{\prime} t} d t \\
=\int_{-\infty}^{+\infty}\left[\sum_{l=1}^{d} a_{m}\left(\theta_{l}, \phi_{l}\right) s_{l}\left(t+\hat{\tau}_{i}^{\prime} / 2\right)+n_{m}(t)\right]
\end{aligned}
$$

$$
\begin{aligned}
& \times s^{H}\left(t-\hat{\tau}_{i}^{\prime} / 2\right) e^{-j 2 \pi \hat{v}_{i}^{\prime} t} d t \\
= & a_{m}\left(\theta_{i}, \phi_{i}\right) \int_{-\infty}^{+\infty} s_{i}\left(t+\hat{\tau}_{i}^{\prime} / 2\right) s^{H}\left(t-\hat{\tau}_{i}^{\prime} / 2\right) e^{-j 2 \pi \hat{v}_{i}^{\prime} t} d t \\
& +\int_{-\infty}^{+\infty} \sum_{l=1, l \neq i}^{d} a_{m}\left(\theta_{l}, \phi_{l}\right) s_{l}\left(t+\hat{\tau}_{i}^{\prime} / 2\right) s^{H}\left(t-\hat{\tau}_{i}^{\prime} / 2\right) e^{-j 2 \pi \hat{v}_{l}^{\prime} t} d t \\
& +\int_{-\infty}^{+\infty} n_{m}(t) s^{H}\left(t-\hat{\tau}_{i}^{\prime} / 2\right) e^{-j 2 \pi \hat{v}_{i}^{\prime} t} d t \\
= & a_{m}\left(\theta_{i}, \phi_{i}\right) \int_{-\infty}^{+\infty} s_{i}\left(t+\hat{\tau}_{i}^{\prime} / 2\right) s^{H}\left(t-\hat{\tau}_{i}^{\prime} / 2\right) e^{-j 2 \pi \hat{v}_{i}^{\prime} t} d t+\beta_{m} . \quad(21)
\end{aligned}
$$

Here, $\beta$ notates for sum of inter-path interference and noise terms. The DOA of the detected multipath component can be estimated from phase of each element of $\mathbf{P}_{\mathbf{i}}$ by optimizing the following criterion:

$$
\begin{aligned}
& \left(\hat{\theta}_{i}, \hat{\phi}_{i}\right) \\
& =\arg \max _{\theta, \phi}\left|\mathbf{a}(\theta, \phi)^{H} \mathbf{P}_{\mathbf{i}}\right| \\
& =\mid \mathbf{a}(\theta, \phi)^{H} \mathbf{a}\left(\theta_{i}, \phi_{i}\right) \int_{-\infty}^{+\infty} s_{i}\left(t+\hat{\tau}_{i}^{\prime} / 2\right) s^{H}\left(t-\hat{\tau}_{i}^{\prime} / 2\right) e^{-j 2 \pi \hat{v}_{i}^{\prime} t} d t \\
& \quad+\sum_{m=1}^{M} a_{m}(\theta, \phi)^{H} \beta_{m} \mid
\end{aligned}
$$

In this cost function, if we concentrate on the first term in the absolute value, accuracy of the maximization procedure is directly related with the accuracy of the integral that is calculated with delay and Doppler estimates $\left(\hat{\tau}_{i}^{\prime}, \hat{v}_{i}^{\prime}\right)$. Therefore, joint estimation approach on incoherently integrated CAF surface not only provides good initial delay and Doppler estimates but also accuracy of DOA estimates are improved. Note that, if there were a single path in the detected delay-Doppler cell, this criterion would have provided highly accurate estimates. Having obtained the DOA of the $i$ th multipath component, in order to further improve the accuracy of the initial delay and Doppler estimates, each antenna output is coherently integrated. To motivate the coherent integration, let's write the output of the $m$ th antenna as:

$$
\begin{aligned}
x_{m}(t) & =\sum_{i=1}^{d} \zeta_{i} s\left(t-\tau_{i}\right) e^{j 2 \pi v_{i} t} a_{m}\left(\theta_{i}, \phi_{i}\right)+n_{m}(t) \\
& =\sum_{i=1}^{d} s_{i}(t) a_{m}\left(\theta_{i}, \phi_{i}\right)+n_{m}(t) \\
& =s_{i}(t) a_{m}\left(\theta_{i}, \phi_{i}\right)+\sum_{l=1, l \neq i}^{d} s_{l}(t) a_{m}\left(\theta_{l}, \phi_{l}\right)+n_{m}(t) .
\end{aligned}
$$

Estimated $\hat{\theta}_{i}$ and $\hat{\phi}_{i}$ values enable coherent combination of individual antenna outputs to obtain $x_{\text {coh }}(t)$ as follows:

$$
\begin{aligned}
x_{\text {coh }}(t) & =\frac{1}{M} \sum_{m=1}^{M} x_{m}(t) a_{m}^{H}\left(\hat{\theta}_{i}, \hat{\phi}_{i}\right) \\
& =\frac{1}{M} \sum_{m=1}^{M}\left[\sum_{i=1}^{d} s_{i}(t) a_{m}\left(\theta_{i}, \phi_{i}\right)+n_{m}(t)\right] a_{m}^{H}\left(\hat{\theta}_{i}, \hat{\phi}_{i}\right)
\end{aligned}
$$




$$
\begin{aligned}
= & s_{i}(t)+\frac{1}{M} \sum_{m=1}^{M} \sum_{l=1, l \neq i}^{d} s_{l}(t) a_{m}\left(\theta_{l}, \phi_{l}\right) a_{m}^{H}\left(\hat{\theta}_{i}, \hat{\phi}_{i}\right) \\
& +\frac{1}{M} \sum_{m=1}^{M} n_{m}(t) a_{m}^{H}\left(\hat{\theta}_{i}, \hat{\phi}_{i}\right) \\
= & s_{i}(t)+\beta_{c o h}(t) .
\end{aligned}
$$

Here, the first term is the sum of phase corrected versions of the $i$ th multipath component at each antenna, second term is the inter-path interference, and the third one is the noise term. If we had one multipath component, inter-path interference would have been zero. Noise power in $x_{\text {coh }}(t)$ can be written as:

$$
\begin{aligned}
\sigma_{\text {coh }}^{2} & =E\left\{\left(\frac{1}{M} \sum_{m=1}^{M} n_{m}(t) a_{m}^{H}\left(\hat{\theta}_{i}, \hat{\phi}_{i}\right)\right)\left(\frac{1}{M} \sum_{k=1}^{M} n_{k}(t) a_{k}^{H}\left(\hat{\theta}_{i}, \hat{\phi}_{i}\right)\right)^{H}\right\} \\
& =\frac{1}{M^{2}} \sum_{m=1}^{M} E\left\{n_{m}(t) n_{k}^{H}(t)\right\} \\
& =\frac{1}{M} \sigma^{2} .
\end{aligned}
$$

Therefore, after coherent integration signal power is unchanged and noise power is reduced by the factor, $1 / M$, in the ideal case. Due to the estimation errors in DOA of the $i$ th multipath component and inter-path interference, reduction in noise variance will not exactly be equal to $1 / M$. However, in each iteration of the CAF-DF algorithm, DOA estimates are updated and inter-path interferences are effectively suppressed. As a result positive effect of the coherent integration in estimation is increased. Therefore, coherent integration process, (24), of the antenna outputs results in an improvement in the SNR. The phase correction procedure, with respect to the array origin, given in (24) is illustrated in Fig. 3, where the slow-time versions (sampled version of the pulse train with pulse repetition interval) of the antenna outputs are seen. If phase shifting with respect to the array origin occurs with the correct DOA estimates, then output slow-time signals overlap as in Fig. 3. As demonstrated in Fig. 2(f), $(\mathrm{h})$, the CAF between the transmitted signal and the signal obtained by coherent integration, $x_{\text {coh }}(t)$,

$$
\begin{aligned}
\chi_{x_{c o h}, s}(\tau, v)= & \int_{-\infty}^{\infty} x_{c o h}(t+\tau / 2) s^{H}(t-\tau / 2) e^{-j 2 \pi v t} d t \\
= & \int_{-\infty}^{\infty} s_{i}(t+\tau / 2) s^{H}(t-\tau / 2) e^{-j 2 \pi v t} d t \\
& +\int_{-\infty}^{\infty} \beta_{c o h}(t+\tau / 2) s^{H}(t-\tau / 2) e^{-j 2 \pi v t} d t
\end{aligned}
$$

yields more accurate detection of delay and Doppler of the detected path than the initial delay and Doppler estimates. In order to visualize the effect of the coherent integration clearly, a 1-D delay slice of the peak point on the incoherently integrated CAF and coherently integrated CAF surfaces, shown in Fig. 2(e)-(h), are presented in Fig. 4. Bold line represents the 1-D slice across the coherently integrated surface and the dashed line represents the $1-D$ slice across the incoherently integrated surface. Note that, interference from other signal paths and the noise is less detrimental around the peak of the coherently integrated CAF. Therefore, the delay-Doppler estimates for the detected path become more accurate. Also note that, in Fig. 4(b), peak location of the incoherently

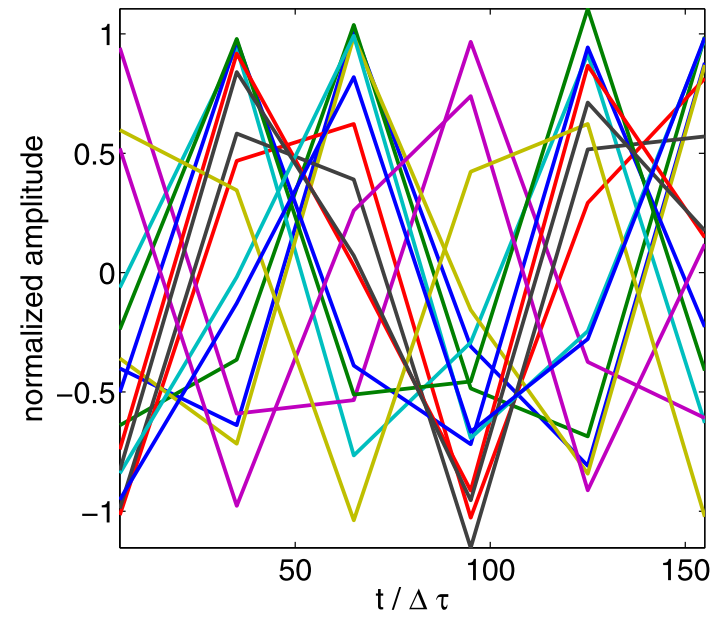

(a)

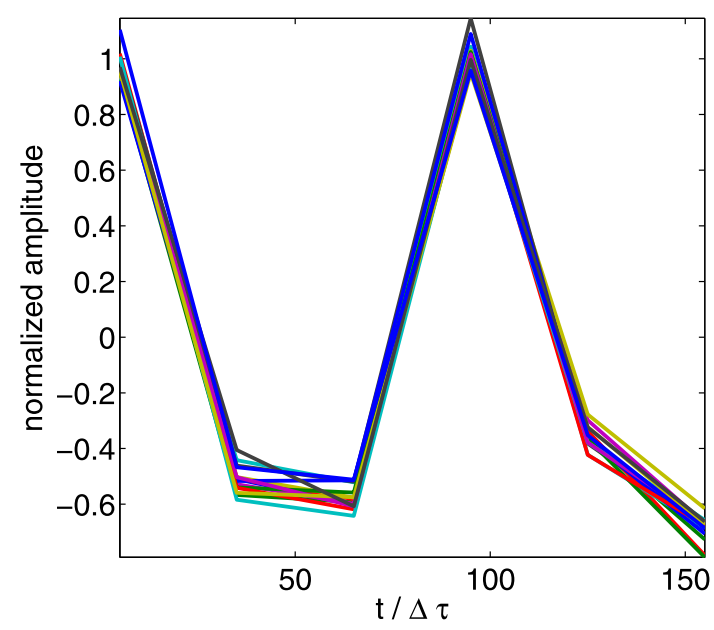

(b)

Fig. 3. Coherent integration process in slow-time for 15-element antenna array. Real part of the complex array output is plotted. (a): Slow-time output of array before coherent integration. (b): Slow-time output of array after coherent integration.

integrated CAF, which is the Doppler estimate of the path-2, is shifted left from its true value. However, peak location of the coherently integrated CAF points the true Doppler value clearly. As a result, initial delay and Doppler estimates $\left(\hat{\tau}_{i}^{\prime}, \hat{v}_{i}^{\prime}\right)$ are updated by calculating the ambiguity function between $x_{\text {coh }}(t)$ and $s(t)$, $\chi_{x_{\text {coh }, s}}(\tau, \nu)$. Delay and Doppler values corresponding to peak of $\chi_{x_{\text {coh }, s}}(\tau, v)$ are considered as updated estimates, $\left(\hat{\tau}_{i}^{\prime \prime}, \hat{v}_{i}^{\prime \prime}\right)$. The obtained estimates for azimuth, elevation, delay and Doppler parameters of one of the impinging paths enables to generate a copy of the impinging signal at each antenna output as:

$\hat{x}_{m, i}(t)=\zeta_{m, i} s\left(t-\hat{\tau}_{i}^{\prime \prime}\right) e^{j 2 \pi \hat{v}_{i}^{\prime \prime} t} e^{-j 2 \pi v_{c} \xi_{m, i}\left(\hat{\theta}_{i}, \hat{\phi}_{i}\right)}$,

where $i$ represents the $i$ th detected signal path and $\zeta_{m, i}$ is a complex scalar, which covers all the phase shifts and attenuation effects and modeled as a uniformly distributed phase between 0 and $2 \pi$. Due to, calibration inaccuracies of the antenna array, $\zeta_{m, i}$ may differ for each antenna. Under additive white Gaussian noise model, conditional maximum likelihood estimate of the $\zeta_{m, i}$ for a given set of estimated $\left(\hat{\tau}_{i}^{\prime \prime}, \hat{v}_{i}^{\prime \prime}, \hat{\theta}_{i}, \hat{\phi}_{i}\right)$ parameters can be obtained as the minimizer of the following cost function:

$$
J_{m}\left(\zeta_{m, i}\right)=\int_{0}^{T_{c o h}}\left|x_{m}(t)-\hat{x}_{m, i}(t)\right|^{2} d t .
$$




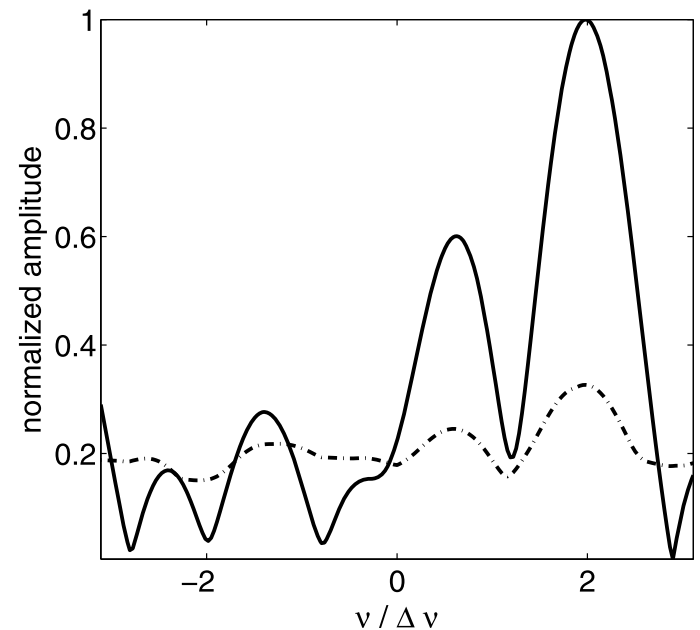

(a)

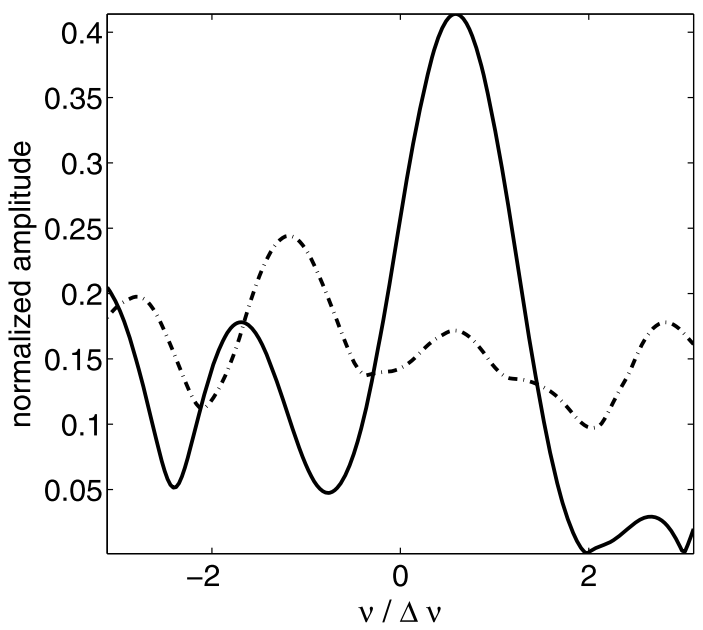

(b)

Fig. 4. Two signal paths with parameters $\tau / \Delta \tau=[1.5,1.5]$ and $\nu / \Delta v=[1.9,0.7]$. 1-D peak-delay slices of CAF surfaces of Fig. 2(e)-(h). Bold and dashed lines are for coherent and incoherent integration, respectively. (a): 1-D peak-delay slice of CAF surface of path-1. (b): 1-D peak-delay slice of CAF surface of path-2.

The least-squares solution of the this quadratic cost function is [43]:

$\hat{\zeta}_{m, i}=\frac{\int_{0}^{T_{c o h}} s^{H}\left(t-\hat{\tau}_{i}^{\prime \prime}\right) e^{-j 2 \pi \hat{v}_{i}^{\prime \prime} t} a_{m}^{H}\left(\hat{\theta}_{i}, \hat{\phi}_{i}\right) x_{m}(t) d t}{\int_{0}^{T_{c o h}} s^{H}\left(t-\hat{\tau}_{i}^{\prime \prime}\right) s\left(t-\hat{\tau}_{i}^{\prime \prime}\right) d t}$.

Note that, if there is negligible calibration issue between the antennas, $\zeta_{m, i}$ will be approximately the same for each antenna and can be estimated optimally as:

$\hat{\zeta}_{i}=\frac{1}{M} \sum_{m=1}^{M} \hat{\zeta}_{m, i}$

Once the complex scaling parameter, $\hat{\zeta}_{m, i}$ is obtained, the identified path is fully characterized. Hence, a copy of the first multipath component at each antenna output can be generated to eliminate it from the array output to recurse on the residual for detection of the remaining multipath components. Although it is in the class of greedy optimization techniques, this iterative approach is highly efficient. Note that, elimination of a multipath component from the array outputs eliminates both its main and sidelobes from the CAF domain. Thus, weaker paths that are buried under the sidelobes of the detected and eliminated path might become detectable as well. An illustration of this fact is shown in Fig. 2, where the CAF of the
Table 1

CAF-DF algorithm.

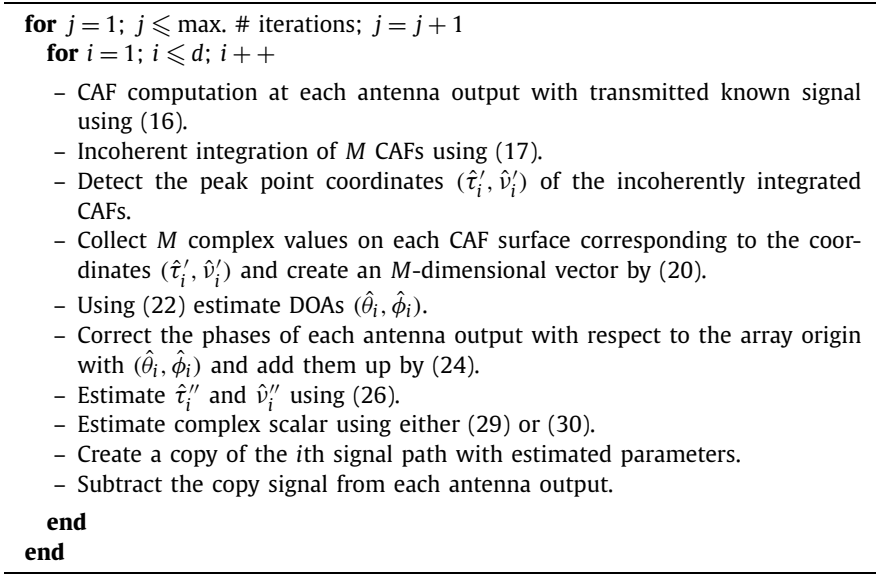

residual array outputs reveals the presence of a weaker path that was partially buried under the sidelobes of the eliminated path. This detection and elimination process is repeated until there is no peak exceeding the detection threshold that can be set to satisfy a constant false alarm rate or a predefined number of iterations. In Table 1, steps of the CAF-DF algorithm are summarized.

\section{Simulation results}

In this section, performances of the CAF-DF, SAGE and MUSIC algorithms are compared on synthetic signals at different SNR values by using Monte Carlo simulations. Implementation details for the MUSIC-based and SAGE techniques are presented in Appendices $A$ and $B$, respectively. The joint-rMSE, the basis of our comparisons, is defined as:

$\mathrm{rMSE}=\sqrt{\frac{1}{d N_{r}} \sum_{i=1}^{d} \sum_{l=1}^{N_{r}}\left[\hat{\boldsymbol{\varphi}}_{i}(l)-\boldsymbol{\varphi}_{i}(l)\right]^{2}}$

where $N_{r}$ is the number of Monte Carlo simulations, $\hat{\varphi}_{i}(l)$ is the parameter estimates of the $i$ th multipath component found in the lth simulation and $\varphi_{i}(l)$ is the true parameter values of the $i$ th path. A circular receiver array of $M$ omnidirectional sensors at positions $[r \cos (m 2 \pi / M), r \sin (m 2 \pi / M)], 1 \leqslant \cdots \leqslant M$, is synthesized. The radius of the array $r=\lambda / 4 \sin (\pi / M)$ is chosen such that the distance between two neighboring sensors is $\lambda / 2$, where $\lambda$ is the carrier wavelength. The transmitted training signal consists of 6 Barker-13 coded pulses with a duration of $13 \Delta \tau$ where $\Delta \tau$ is the chip duration. The pulse repetition interval is $30 \Delta \tau$ resulting a total signal duration of $q T=167 \Delta \tau$. The SNR is defined at a single sensor relative to the noise variance. Both the CAF-DF and the SAGE algorithms are iterated only 4 times, which is found to be sufficient for convergence. All simulations are conducted on a DELL Latitude E6410 Laptop with Intel-i7 $2.8 \mathrm{GHz}$ processor. Single iteration of the CAF-DF and the SAGE techniques for one multipath component take approximately $0.73 \mathrm{~s}$ and $0.97 \mathrm{~s}$, respectively. Proposed technique is computationally efficient than the SAGE technique. The MUSIC technique is known to be computationally very efficient technique and it takes $0.2 \mathrm{~s}$ for one multipath component.

In the first experiment there exist two equal power paths having parameters $\varphi_{1}=\left[50^{\circ}, 40^{\circ}, 2 \Delta \tau, 1.7 \Delta \nu, e^{j \psi_{1}}\right]$ and $\varphi_{2}=$ $\left[54^{\circ}, 44^{\circ}, 1.5 \Delta \tau, 0.8 \Delta \nu, e^{j \psi_{2}}\right]$, where $\psi_{1}$ and $\psi_{2}$ are uniformly distributed between $[0,2 \pi]$. A uniform circular array of $M=15$ sensors is used. Note that, the two paths are closely spaced in all parameters. Time-delay and Doppler shift difference between 


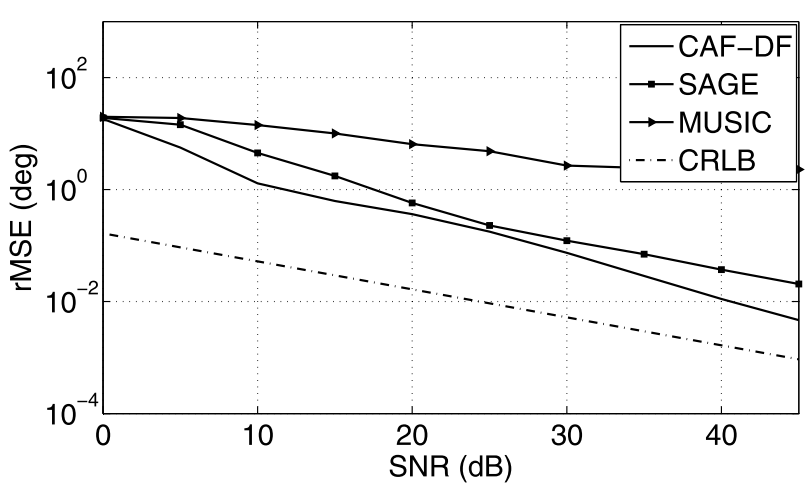

(a)

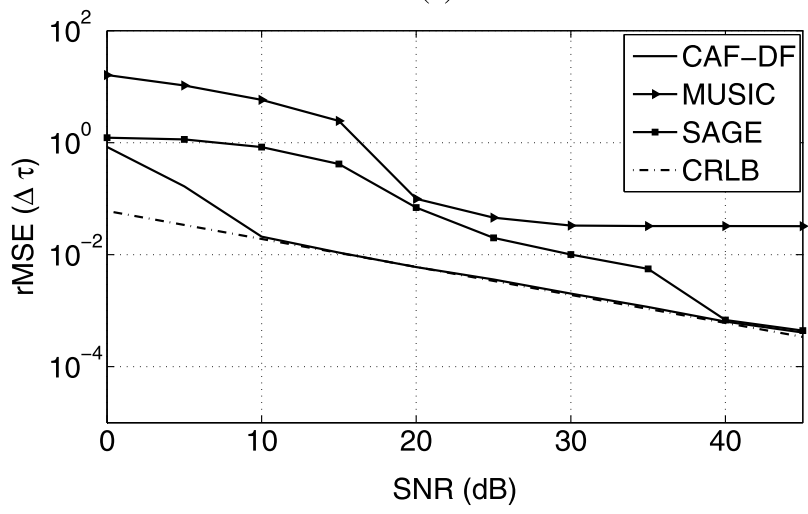

(c)

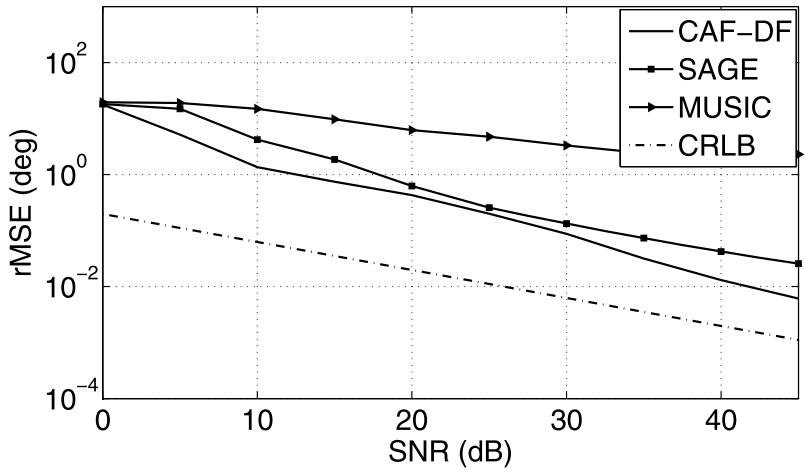

(b)

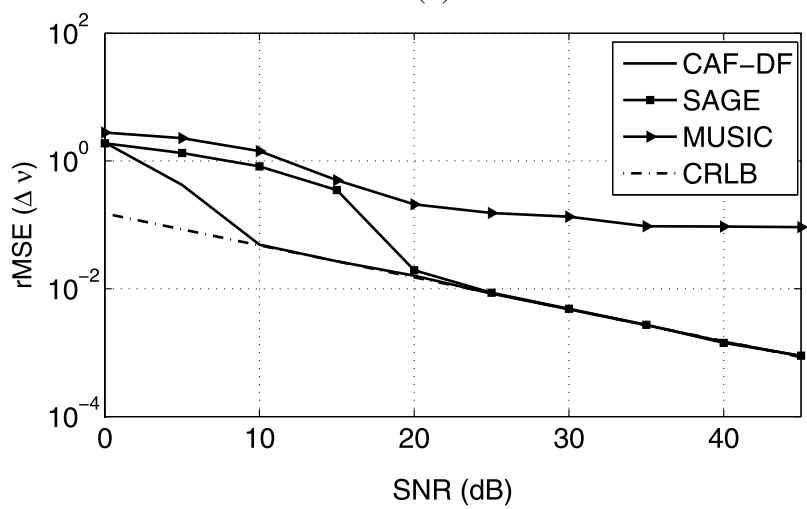

(d)

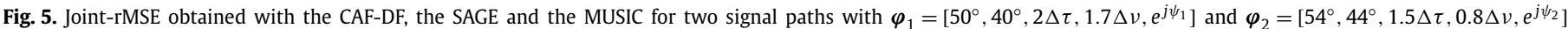
at different SNR values. (a): azimuth, (b): elevation, (c): time-delay and (d): Doppler shift.

the two paths are $0.5 \Delta \tau$ and $0.9 \Delta v$ respectively in CAF domain. Two paths are $4^{\circ}$ separated in spatial domain. Fig. 5 presents the rMSE obtained from 500 Monte Carlo runs at each SNR. Time-delay and Doppler rMSEs are normalized by $\Delta \tau$ and $\Delta v$, respectively. Obtained results show that both the CAF-DF and the SAGE techniques provide significantly better parameter estimates than the MUSIC technique. Furthermore, the CAF-DF technique outperforms the SAGE technique and provides more reliable estimates at all simulated SNR values. Since in many applications low SNR performance is a deciding factor, the superior performance of the CAFDF at low SNRs is a significant improvement. Delay and Doppler parameter estimates of the CAF-DF algorithm achieved the CRLB bound. However, for the considered SNR values in the simulation, DOA estimates could not achieved the lower bound. We think that, this is due to the separate and suboptimal approach used in the iterations of the algorithm. Using the same settings of the first experiment, Fig. 6 illustrates the convergence of rMSE for the CAF-DF and the SAGE techniques at $30 \mathrm{~dB}$ SNR. As expected, the rMSE of each parameter has a monotonic decrease with iterations. Both algorithms converge in a few iterations.

In the second simulation study, there exist two equal power paths with parameters $\varphi_{1}=\left[50^{\circ}, 40^{\circ}, 1.5 \Delta \tau, 1.6 \Delta \nu, e^{j \psi_{1}}\right]$ and $\boldsymbol{\varphi}_{2}=\left[54^{\circ}, 44^{\circ}, 1.66 \Delta \tau, 0.8 \Delta v, e^{j \psi_{2}}\right]$. A uniform circular array with $M=15$ sensors is used. This time the paths are even more closer. Time-delay and Doppler shift difference between the two paths are $0.16 \Delta \tau$ and $0.8 \Delta v$, respectively. Paths can now be separated only by using the difference in their Doppler shift. The obtained results are tabulated in Table 2. As in the first experiment, MUSIC is not able to separate the paths. Performances of the CAF-DF and the SAGE are degraded slightly, as expected. In this scenario, except the Doppler estimates at high SNRs, the CAFDF consistently performs better than the SAGE.
Table 2

rMSE values of MUSIC (A1), SAGE (A2) and CAF-DF (A3) algorithms for various SNR values. CRLB (A4). Time-delay and Doppler rMSEs are normalized by $\Delta \tau$ and $\Delta \nu$, respectively.

\begin{tabular}{lccccl}
\hline \multicolumn{2}{l}{ SNR $(\mathrm{dB})$} & rMSE $(\mathrm{deg})$ & rMSE $(\mathrm{deg})$ & $\mathrm{rMSE} / \Delta \tau$ & $\mathrm{rMSE} / \Delta \nu$ \\
\hline 10 & A1 & 15.58 & 15.79 & 11.84 & 1.56 \\
& A2 & 4.23 & 3.7 & 0.9 & 1.1 \\
& A3 & 1.73 & 2.45 & 0.07 & 0.46 \\
& A4 & 0.02 & 0.027 & 0.019 & 0.046 \\
15 & A1 & 14.48 & 14.24 & 11.62 & 1.42 \\
& A2 & 2.07 & 1.91 & 0.44 & 0.76 \\
& A3 & 0.83 & 0.84 & 0.01 & 0.051 \\
& A4 & 0.011 & 0.016 & 0.01 & 0.025 \\
20 & A1 & 12.32 & 14.06 & 11.48 & 1.3 \\
& A2 & 0.61 & 0.66 & 0.077 & 0.035 \\
& A3 & 0.41 & 0.49 & 0.0068 & 0.025 \\
& A4 & 0.006 & 0.0092 & 0.0061 & 0.014 \\
25 & A1 & 11.75 & 12.68 & 10.2 & 1.04 \\
& A2 & 0.22 & 0.25 & 0.019 & 0.013 \\
& A3 & 0.22 & 0.27 & 0.0037 & 0.016 \\
& A4 & 0.0036 & 0.0052 & 0.0034 & 0.008 \\
30 & A1 & 10.6 & 10.18 & 8.2 & 0.63 \\
& A2 & 0.12 & 0.12 & 0.011 & 0.006 \\
& A3 & 0.08 & 0.11 & 0.0024 & 0.012 \\
& A4 & 0.002 & 0.0026 & 0.0019 & 0.004 \\
\hline
\end{tabular}

In the third experiment, we investigated the identification of 10 paths with a circular array of 8 sensors. The path parameters are given in Table 3. The delay-Doppler domain spread of these paths are shown in Fig. 7. Note that the number of paths exceeds the number of sensors which would made it impossible to resolve with narrowband systems. However, in wideband communication systems, delay-Doppler domain diversity of the paths can be exploited to resolve the paths as long as there are 


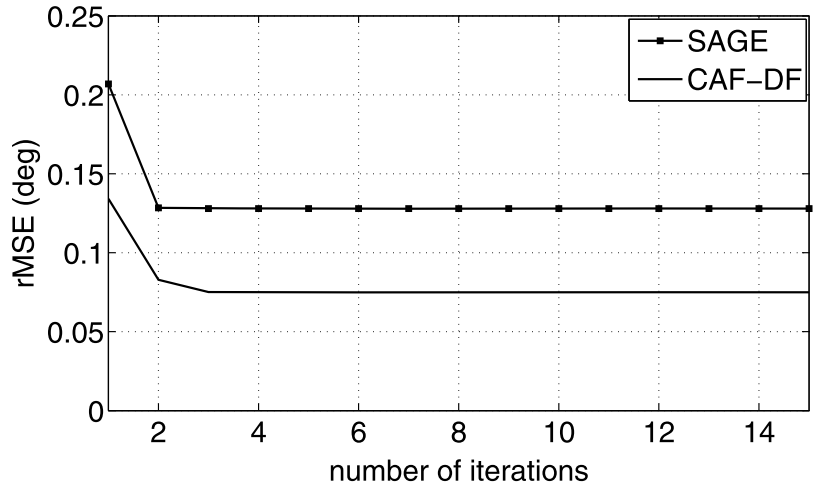

(a)

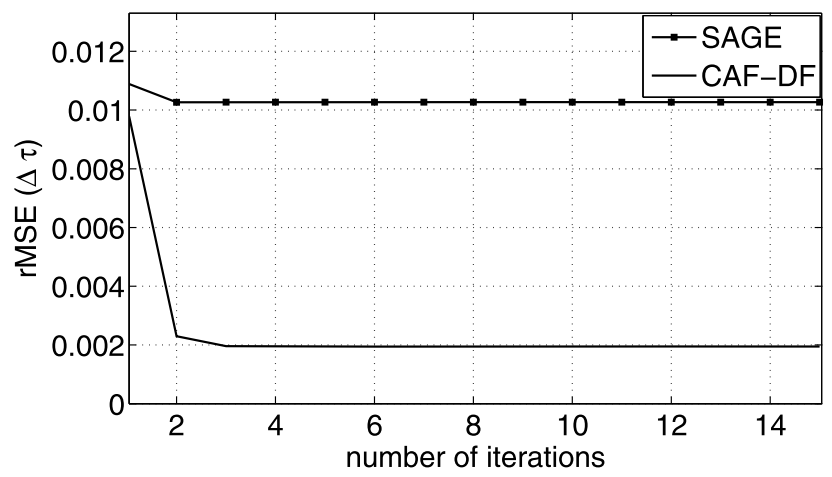

(c)

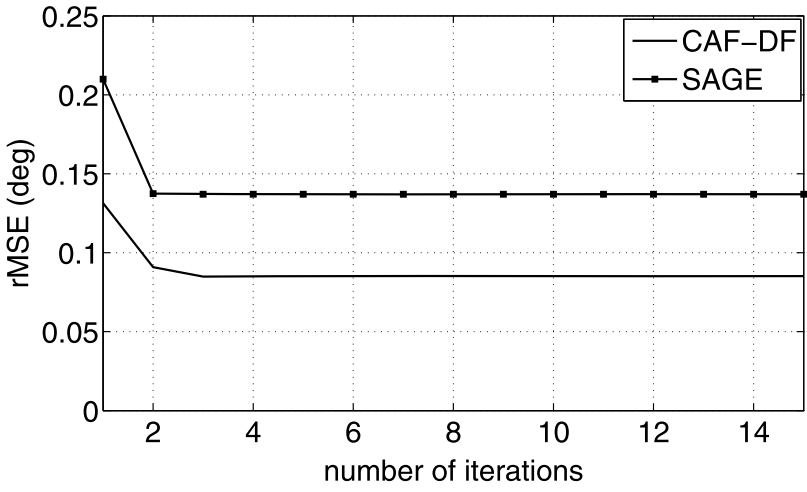

(b)

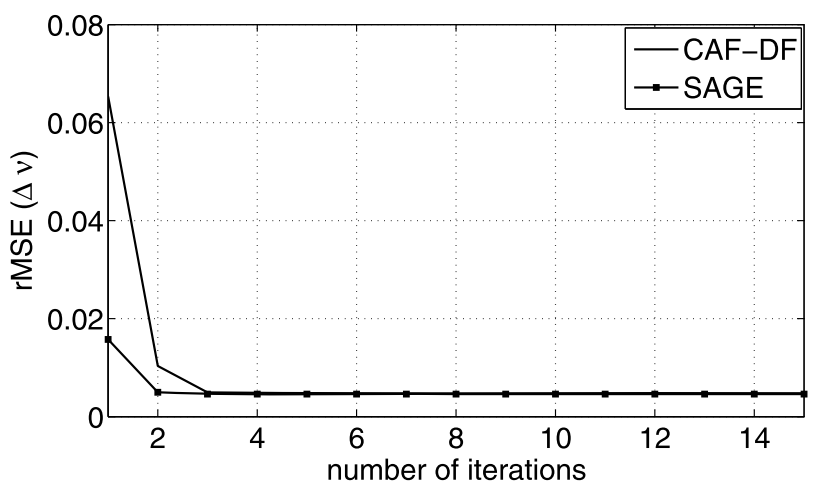

(d)

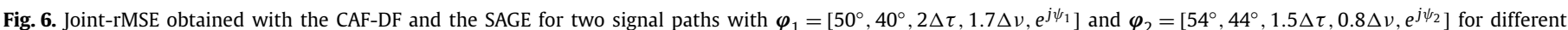
number of iterations. (a): azimuth, (b): elevation, (c): time-delay and (d): Doppler shift.

Table 3

10 path parameters. Time-delay, Doppler and complex scaling factor values are normalized by $\Delta \tau, \Delta v$ and $e^{j \psi_{i}}$, respectively. $\psi_{i}$ 's, $i=1, \ldots, d$, are uniformly distributed between $[0,2 \pi]$.

\begin{tabular}{clllll}
\hline Path & $\theta(\mathrm{deg})$ & $\phi(\mathrm{deg})$ & $\tau / \Delta \tau$ & $\nu / \Delta \nu$ & $\zeta / e^{j \psi_{i}}$ \\
\hline 1 & 45 & 30 & 1 & 1 & 1 \\
2 & 50 & 35 & 1.66 & 1.5 & 0.9 \\
3 & 55 & 40 & 1.16 & 2.5 & 0.8 \\
4 & 60 & 45 & 1.83 & 3 & 0.7 \\
5 & 65 & 50 & 2.5 & 2.7 & 0.6 \\
6 & 70 & 55 & 3.16 & 3.4 & 0.8 \\
7 & 75 & 38 & 4.16 & 1.6 & 0.8 \\
8 & 57 & 47 & 4.83 & 1 & 1 \\
9 & 63 & 43 & 4.66 & 2.8 & 1 \\
10 & 68 & 33 & 5.5 & 2.1 & 0.7 \\
\hline
\end{tabular}

fewer paths than the number of array elements in each resolvable delay-Doppler cell. The joint-rMSE of the estimated multipath parameters by the proposed CAF-DF and the SAGE algorithms are shown in Fig. 8. We observed that the CAF-DF technique provided significantly better estimates at all SNR levels. The main reason for failure of the SAGE is that, when the number of paths increases, the maximum likelihood-based approach faces significant challenges in finding the global maximum of the likelihood function. This is mainly because of the fact that likelihood maximization is performed in time domain, where there is a considerable overlap between the signals received from different paths. However, CAF-DF technique transforms the array signal outputs to the CAF domain where different multipath signals are localized to their respective delay and Doppler cell. Therefore, overlapping signals in time domain are separated in delay-Doppler domain resulting in the observed performance improvement. Moreover, in Fig. 9,

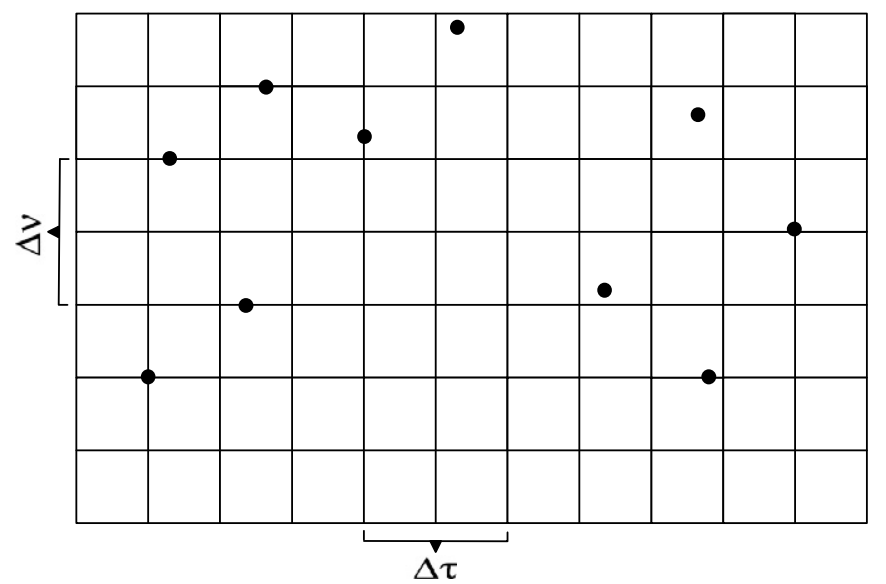

Fig. 7. Delay-Doppler spread of the 10 signal paths are represented with black dots on the delay-Doppler domain.

$\widehat{S N R}_{\text {CAF-DF }} / \widehat{S N R}_{\text {SAGE }}$ ratio is plotted for threshold and asymptotic regions of estimation performance at various SNR values using Eqs. (12) and (15). Parallel with the results shown in Fig. 8, at all SNR levels CAF-DF combines diversity better than the SAGE which enable detector to accurately retrieve the transmitted information.

\section{Conclusions}

A new array signal processing technique, called as CAF-DF, is proposed for the estimation of multipath channel parameters including the path amplitude, delay, Doppler shift and DOAs. The 


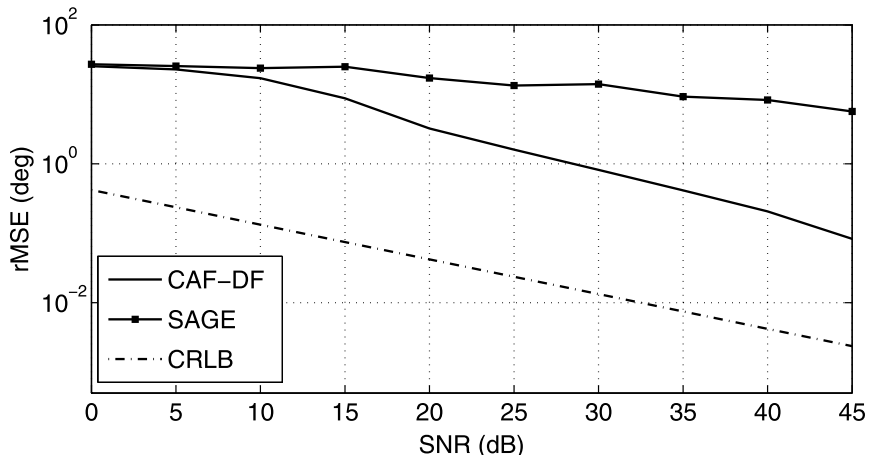

(a)

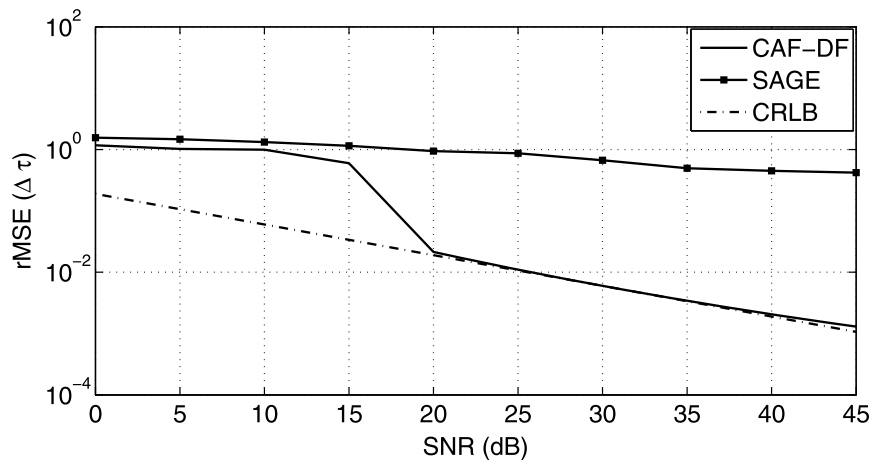

(c)

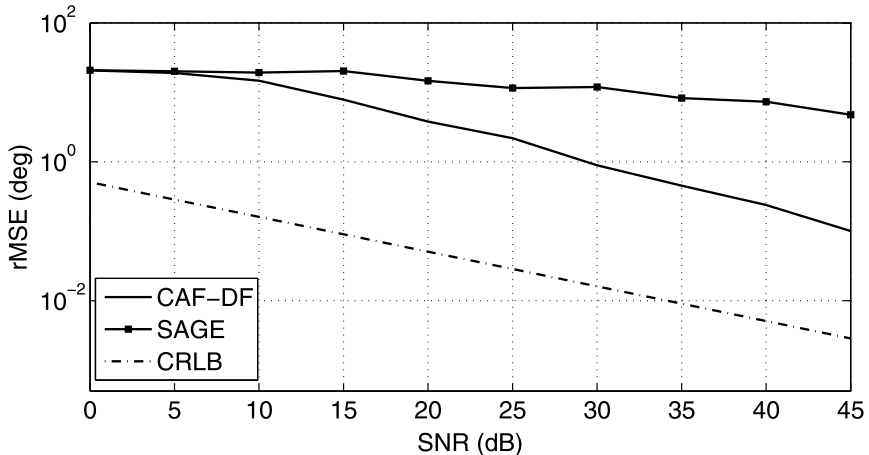

(b)

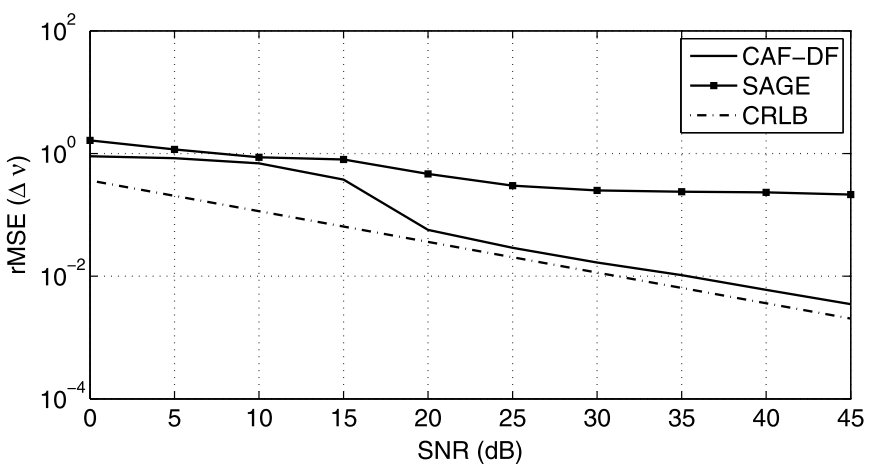

(d)

Fig. 8. Joint-rMSE, obtained with the CAF-DF and the SAGE for 10 signal paths at different SNR values. (a): azimuth, (b): elevation, (c): time-delay and (d): Doppler shift.

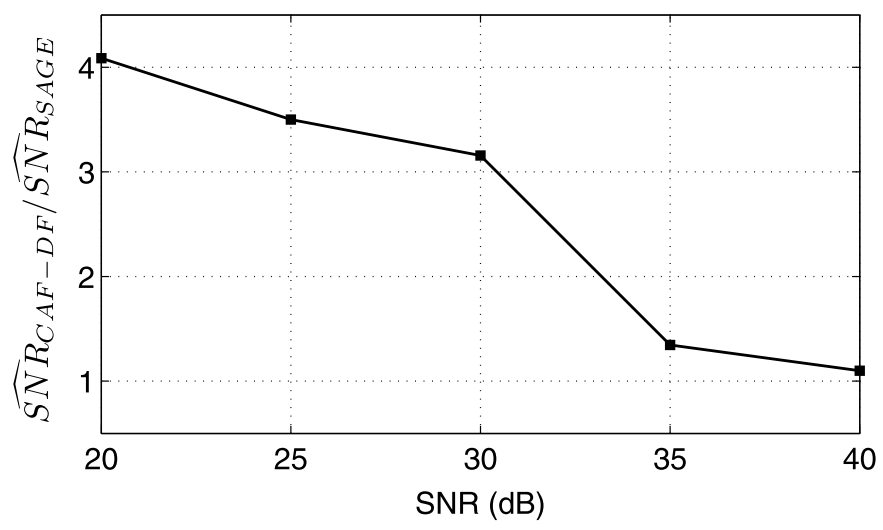

Fig. 9. Ratio of estimated SNRs of CAF-DF and SAGE techniques for threshold and asymptotic regions of estimation performance using (12), (15).

proposed CAF-DF technique makes use of the CAF computation for joint and reliable estimation of path parameters of individual multipath components. Extensive simulation results show that the CAF-DF technique is superior in terms of rMSE to the SAGE technique over a wide range of SNR levels. Furthermore, the CAFDF technique provides 2 to $3 \mathrm{~dB}$ improvement over the SAGE technique in the SNR of diversity combined detection signal. This improvement provided over the practical operational SNR range of receivers is a very significant advantage of the CAF-DF technique.

\section{Acknowledgment}

We would like to thank Dr. Sinan Gezici for valuable discussion.

\section{Appendix A. MUSIC-based algorithm}

MUSIC (MUltiple SIgnal Classification) is a widely used signal subspace method which finds the array manifold orthogonal to the noise subspace. The MUSIC spectrum is computed by performing an eigenvalue analysis on the correlation matrix. The space spanned by the eigenvectors consists of two disjoint subspaces: signal and noise subspaces. Eigenvalue decomposition of the covariance matrix in (10) can be expressed as

$\mathbf{R}^{S}=\mathbf{U}_{s} \boldsymbol{\Lambda}_{s} \mathbf{U}_{s}^{H}+\mathbf{U}_{n} \boldsymbol{\Lambda}_{n} \mathbf{U}_{n}^{H}$,

where $\mathbf{U}_{s}$ is the matrix whose columns are the eigenvectors corresponding to the $d$ largest eigenvalues of $\mathbf{R}^{s}$, and $\mathbf{U}_{n}$ is an $N \times$ $(N-d)$ matrix whose columns are rest of the eigenvectors of $\mathbf{R}^{s}$. Column spaces of $\mathbf{U}_{s}$ and $\mathbf{U}_{n}$ span the signal and noise subspaces respectively. Columns of $\mathbf{U}_{n}$ are orthogonal to $\mathbf{U}_{s}$. In order to estimate the DOAs, one should determine $d$ values of $(\theta, \phi)$ set that make $\mathbf{a}(\theta, \phi)$ nearly orthogonal to $\left(\mathbf{I}-\mathbf{U}_{s} \mathbf{U}_{s}^{H}\right)$, according to

$\mathcal{P}(\theta, \phi)=\frac{\mathbf{a}^{H}(\theta, \phi) \mathbf{a}(\theta, \phi)}{\mathbf{a}^{H}(\theta, \phi)\left(\mathbf{I}-\mathbf{U}_{s} \mathbf{U}_{s}^{H}\right) \mathbf{a}(\theta, \phi)}$.

Having estimated the DOAs, delay and Doppler parameters are estimated. For that purpose, each antenna output is correlated with training coded pulse, $p(t)$ as follows:

$\mathbf{X}_{c}=\operatorname{corr}(\mathbf{X}, p(t))$.

Then, in order to compress noise a simple averaging scheme is applied. To do this, a $q \times 1$ vector is created at each antenna output by taking samples that are periodic with $T$;

$\mathbf{C}_{m}^{n}=\left[\mathbf{X}^{c}\left(n . t_{s}+T\right), \ldots, \mathbf{X}^{c}\left(\text { n.t }_{s}+q T\right)\right]^{T}$,
$m=1,2, \ldots, M$,

where $t_{s}$ is the sampling time and $n=1 / t_{s}, \ldots, T / t_{s}$. By adding up norm-squared $\mathbf{C}_{m}^{n}$ values, we simply get

$\kappa[n]=\frac{1}{M} \sum_{m=1}^{M}\left\|C_{m}^{n}\right\|^{2}$. 
Dominant peak points of $\kappa[n]$ correspond to probable signal arrival paths. By setting a threshold value, locations of the dominant peaks are obtained. Time domain pair of the indexes are the delay estimates of paths. For each peak, corresponding correlated antenna output vectors, $C_{m}^{n}$, are merged to form an $M \times\left(T / t_{s}\right)$ matrix. Using this matrix as an input to the classical MUSIC algorithm, DOAs of the each signal source are found [4]. As discussed in coherent integration step of the CAF-DF technique, in order to increase the SNR, with the estimated DOAs each antenna correlated output is phase corrected with respect to the origin of the array and added up. Lastly, Fourier transform of the resultant vector is evaluated to find the Doppler shift of the impinging signal source.

A limitation of the MUSIC occurs when two or more sources are highly correlated, which is the scenario considered in the simulations. Contrary to this bottleneck, for accurate signal models and in medium-high SNR conditions, the MUSIC is a statistically consistent, computationally very efficient and well studied technique in the literature [2]. Moreover, due to its ease of implementation and computational efficiency, it has been used in many different realtime applications successfully [6,7]. Therefore, since it is a very well-known technique, we wanted to include its relative performance to the CAF-DF and the SAGE techniques in two different channel estimation scenarios.

\section{Appendix B. The SAGE algorithm}

One of the most popular approaches to obtain more efficient ML estimates is the EM algorithm [18]. EM is an iterative method for solving the ML estimation problem in situations where a part of the observations are missing. To further improve the speed of convergence of the EM approach, SAGE algorithm has been proposed [22]. Each iteration of the algorithm contains EM iteration phase where some of the parameters are fixed at the previous iteration values, while other parameters are re-estimated. Instead of simultaneous parameter estimation, parameters are estimated sequentially. In order to reduce the complexity of the algorithm, suboptimal but faster one-dimensional optimization procedures along each parameter are used. In Table 4, the basic form of the SAGE algorithm is presented [25]. Formulization of the SAGE algorithm relies on two crucial points of unobservable (complete) and observable (incomplete) data. Considering the model given in (5), complete data can be defined as follows:

$$
\begin{aligned}
\mathbf{z}_{i}(t) & =\zeta_{i} \mathbf{a}\left(\theta_{i}, \phi_{i}\right) s\left(t-\tau_{i}\right) e^{j 2 \pi v_{i} t}+\mathbf{n}_{i}(t) \\
& =\mathbf{u}_{i}(t)+\mathbf{n}_{i}(t), \quad i=1, \ldots, i, \ldots, d .
\end{aligned}
$$

The received signal is called as incomplete data and it is related to complete data by

$\mathbf{x}(t)=\sum_{i=1}^{d} \mathbf{z}_{i}(t)$

The SAGE algorithm can be divided into two parts namely: expectation and maximization phases. In the expectation phase, complete data can be formed as:

$\hat{\mathbf{z}}_{i}(t ; \eta)=\mathbf{x}(t)-\sum_{\gamma=1, \gamma \neq i}^{d} \hat{\mathbf{u}}\left(t ; \varphi_{i}(\eta)\right)$

where $\eta$ is the algorithm iteration index. In the first iteration, $\eta=1, \hat{\mathbf{z}}_{i}(t ; \eta)$ is initialized as $\hat{\mathbf{z}}_{i}(t ; \eta)=\mathbf{x}(t)$. Once the complete information is formed, the maximization phase takes place to yield a new set of parameter estimates for each multipath component by using the following equations:
Table 4

Basic SAGE algorithm for reference.

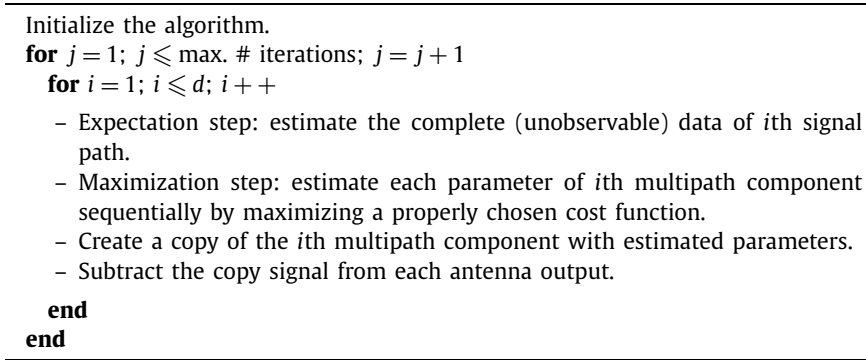

$$
\begin{aligned}
& \hat{\tau}_{i}(\eta) \\
& =\arg \max _{\tau}\left\{\left|\mathbf{g}_{i}\left(\tau, \hat{\theta}_{i}(\eta-1), \hat{\phi}_{i}(\eta-1), \hat{v}_{i}(\eta-1) ; \hat{\mathbf{z}}_{i}(t ; \eta-1)\right)\right|\right\},
\end{aligned}
$$

$\hat{v}_{i}(\eta)=\arg \max _{v}\left\{\left|\mathbf{g}_{i}\left(\hat{\tau}_{i}(\eta), v, \hat{\phi}_{i}(\eta-1), \hat{\theta}_{i}(\eta-1) ; \hat{\mathbf{z}}_{i}(t ; \eta-1)\right)\right|\right\}$,

$\hat{\theta}_{i}(\eta), \hat{\phi}_{i}(\eta)=\arg \max _{\theta, \phi}\left\{\left|\mathbf{g}_{i}\left(\hat{\tau}_{i}(\eta), \theta, \phi, \hat{v}_{i}(\eta) ; \hat{\mathbf{z}}_{i}(t ; \eta-1)\right)\right|\right\}$,

$\hat{\zeta}_{i}(\eta)=\frac{\mathbf{g}_{i}\left(\hat{\tau}_{i}(\eta), \hat{\theta}_{i}(\eta), \hat{\phi}_{i}(\eta), \hat{v}_{i}(\eta) ; \hat{\mathbf{z}}_{i}(t ; \eta-1)\right)}{s(t)^{H} s(t)\left\|\mathbf{a}\left(\hat{\theta}_{i}(\eta), \hat{\phi}_{i}(\eta)\right)\right\|^{2}}$.

In these equations, $\mathbf{g}_{i}\left(\tau, \theta, \phi, \nu ; \mathbf{z}_{i}(t)\right)$ is defined as:

$\mathbf{g}_{i}\left(\tau, \theta, \phi, \nu ; \mathbf{z}_{i}(t)\right) \triangleq \int_{-\infty}^{\infty} s^{H}(t-\tau) \mathbf{a}^{H}(\theta, \phi) \mathbf{z}_{i}(t) e^{-j 2 \pi v t} d t$.

There are various methods to initialize the algorithm. In this paper, the following initialization procedure is preferred. Since, initially, phases of the complex amplitudes $\zeta_{m_{i}}$ are not known, delays and Doppler shifts are estimated incoherently. For this purpose, in the initialization part, maximization procedures used for delay and Doppler estimations given in (B.4) and (B.5) are changed with the equations below.

$\hat{\tau}_{i}(\eta)=\arg \max _{\tau}\left\{\sum_{m=1}^{M}\left|\int_{-\infty}^{\infty} s^{H}(t-\tau) \hat{z}_{m, i}(t, 0) d t\right|\right\}$,

$\hat{v}_{i}(\eta)=\arg \max _{v}\left\{\sum_{m=1}^{M}\left|\int_{-\infty}^{\infty} s^{H}\left(t-\hat{\tau}_{i}(\eta)\right) \hat{z}_{m, i}(t, 0) e^{-j 2 \pi v t} d t\right|\right\}$.

As seen from the equations above, signal estimates for the multipaths with initialized parameters are subtracted from the observed data $\mathbf{x}(t)$. Parameter update procedure is continued until there is no considerable improvement in the sense of rMSE between consecutive iterations.

\section{Appendix C. THE Cramér-Rao bound}

In this appendix, derivation of the CRLB for the joint estimation problem is presented. CRLB provides a lower bound on the variance of the parameter estimates of an unbiased estimator [43]:

$E\left\{(\hat{\boldsymbol{\varphi}}-\boldsymbol{\varphi})^{T}(\hat{\boldsymbol{\varphi}}-\boldsymbol{\varphi})\right\} \geqslant \mathbf{J}^{-1}$,

where $\boldsymbol{\varphi}$ is the parameter vector and $\mathbf{J}$ is the Fisher Information Matrix (FIM). In our model, residual error vector $\mathbf{e}\left(t_{k}\right)$ defined below has a circularly symmetric i.i.d. Gaussian distribution: 
$\mathbf{e}\left(t_{k}\right)=\mathbf{x}\left(t_{k}\right)-\sum_{i=1}^{d} \mathbf{a}\left(\theta_{i}, \phi_{i}\right) \zeta_{i} s\left(t_{k}-\tau_{i}\right) e^{j 2 \pi v_{i} t}$

Assuming the variance of the distribution is $\sigma^{2}$, the log likelihood function can be written as

$\mathfrak{L}=-N M \log \pi \sigma^{2}-\frac{1}{\sigma^{2}} \sum_{k=1}^{N}\left\|\mathbf{e}\left(t_{k}\right)\right\|^{2}$.

Using straight forward differentiations we obtain the following partial differentials that will be used to derive the entries of the FIM:

$\frac{\partial \mathfrak{L}}{\partial \tau_{i}}=\frac{2}{\sigma^{2}} \sum_{k=1}^{N} \Re e\left[\zeta_{i}^{H} \mathbf{a}^{H}\left(\theta_{i}, \phi_{i}\right) e^{-j 2 \pi} v_{i} t_{k} \frac{\partial S^{H}\left(t_{k}-\tau_{i}\right)}{\partial t} \mathbf{e}\left(t_{k}\right)\right]$

$\frac{\partial \mathfrak{L}}{\partial \theta_{i}}=\frac{2}{\sigma^{2}} \sum_{k=1}^{N} \Re e\left[\zeta_{i}^{H} s^{H}\left(t_{k}-\tau_{i}\right) e^{-j 2 \pi v_{i} t_{k}} \frac{\partial \mathbf{a}^{H}\left(\theta_{i}, \phi_{i}\right)}{\partial \theta_{i}} \mathbf{e}\left(t_{k}\right)\right]$,

$\frac{\partial \mathfrak{L}}{\partial \phi_{i}}=\frac{2}{\sigma^{2}} \sum_{k=1}^{N} \Re e\left[\zeta_{i}^{H} s^{H}\left(t_{k}-\tau_{i}\right) e^{-j 2 \pi v_{i} t_{k}} \frac{\partial \mathbf{a}^{H}\left(\theta_{i}, \phi_{i}\right)}{\partial \phi_{i}} \mathbf{e}\left(t_{k}\right)\right]$

$\frac{\partial \mathfrak{L}}{\partial v_{i}}=\frac{-4 \pi}{\sigma^{2}} \sum_{k=1}^{N} t_{k} \Im m\left[\zeta_{i}^{H} s^{H}\left(t_{k}-\tau_{i}\right) e^{-j 2 \pi} v_{i} t_{k} \mathbf{a}^{H}\left(\theta_{i}, \phi_{i}\right) \mathbf{e}\left(t_{k}\right)\right]$.

If we define $\zeta_{i}=\eta_{i}+j \kappa_{i}$, we obtain

$$
\begin{aligned}
& \frac{\partial \mathfrak{L}}{\partial \eta_{i}}=\frac{2}{\sigma^{2}} \sum_{k=1}^{N} \Re e\left[s^{H}\left(t_{k}-\tau_{i}\right) e^{-j 2 \pi v_{i} t_{k}} \mathbf{a}^{H}\left(\theta_{i}, \phi_{i}\right) \mathbf{e}\left(t_{k}\right)\right], \\
& \frac{\partial \mathfrak{L}}{\partial \kappa_{i}}=\frac{2}{\sigma^{2}} \sum_{k=1}^{N} \Im m\left[s^{H}\left(t_{k}-\tau_{i}\right) e^{-j 2 \pi v_{i} t_{k}} \mathbf{a}^{H}\left(\theta_{i}, \phi_{i}\right) \mathbf{e}\left(t_{k}\right)\right] .
\end{aligned}
$$

The following identities [44], are useful to determine the elements of the FIM.

$$
\begin{aligned}
& E\left[\boldsymbol{e}_{i}\left(t_{k}\right) \boldsymbol{e}_{c}^{H}\left(t_{j}\right)\right]=\delta_{i c} \delta_{t_{k} t_{j}} \sigma^{2}, \\
& E\left[\boldsymbol{e}_{i}\left(t_{k}\right) \boldsymbol{e}_{z}\left(t_{j}\right)\right]=0, \\
& E\left[\boldsymbol{e}_{c}^{H}\left(t_{k}\right) \boldsymbol{e}_{l}\left(t_{j}\right) \boldsymbol{e}_{n}\left(t_{i}\right)\right]=0, \\
& E\left[\boldsymbol{e}\left(t_{k}\right) \boldsymbol{e}^{H}\left(t_{k}\right) \boldsymbol{e}\left(t_{j}\right) \boldsymbol{e}^{H}\left(t_{j}\right)\right]=M^{2} \sigma^{4}+\delta_{t_{k} t_{j}} M \sigma^{4},
\end{aligned}
$$

where $\boldsymbol{e}_{i}\left(t_{k}\right)$ is the $i$ th component of the $\boldsymbol{e}\left(t_{k}\right)$ and $\delta$ represents Kronecker's delta. Moreover, to make equations shorter, the following equalities are defined:

$R_{s^{\prime} s}^{i, l}(k)=\frac{\partial s^{H}\left(t_{k}-\tau_{i}\right)}{\partial t} s\left(t_{k}-\tau_{l}\right)$,

$\gamma(k)=e^{-j 2 \pi\left(\nu_{i}-v_{l}\right) t_{k}}$,

$\Psi_{\mathbf{a}_{\theta}^{\prime} \mathbf{a}}^{i, l}=\frac{\partial \mathbf{a}^{H}\left(\theta_{i}, \phi_{i}\right)}{\partial \theta_{i}} \mathbf{a}\left(\theta_{l}, \phi_{l}\right)$.

Using these identities elements of the FIM matrix can be obtained as follows:

$E\left[\frac{\partial \mathfrak{L}}{\partial \tau_{i}} \frac{\partial \mathfrak{L}}{\partial \tau_{l}}\right]=\frac{2}{\sigma^{2}} \Re e\left[\Psi_{\mathbf{a a}}^{i, l} \zeta_{i}^{H} \zeta_{l} \sum_{k=1}^{N} R_{s^{\prime} s^{\prime}}^{i, l}(k) \gamma(k)\right]$.

Similarly, other FIM elements can be obtained as:

$$
\begin{aligned}
& E\left[\frac{\partial \mathfrak{L}}{\partial \theta_{i}} \frac{\partial \mathfrak{L}}{\partial \theta_{l}}\right]=\frac{2}{\sigma^{2}} \Re e\left[\zeta_{i}^{H} \zeta_{l} \Psi_{\mathbf{a}_{\theta} \mathbf{a}_{\theta}^{\prime}}^{i, l} \sum_{k=1}^{N} R_{s S}^{i, l}(k) \gamma(k)\right], \\
& E\left[\frac{\partial \mathfrak{L}}{\partial \phi_{i}} \frac{\partial \mathfrak{L}}{\partial \phi_{l}}\right]=\frac{2}{\sigma^{2}} \Re e\left[\zeta_{i}^{H} \zeta_{l} \Psi_{\mathbf{a}_{\phi}^{\prime} \mathbf{a}_{\phi}^{\prime}}^{i, l} \sum_{k=1}^{N} R_{s S}^{i, l}(k) \gamma(k)\right], \\
& E\left[\frac{\partial \mathfrak{L}}{\partial v_{i}} \frac{\partial \mathfrak{L}}{\partial v_{l}}\right]=\frac{8 \pi^{2}}{\sigma^{2}} \Re e\left[\Psi_{\mathbf{a} a}^{i, l} \zeta_{i}^{H} \zeta_{l} \sum_{k=1}^{N} R_{s S}^{i, l}(k) \gamma(k) t_{k}^{2}\right], \\
& E\left[\frac{\partial \mathfrak{L}}{\partial \eta_{i}} \frac{\partial \mathfrak{L}}{\partial \eta_{l}}\right]=\frac{2}{\sigma^{2}} \Re e\left[\Psi_{\text {aa }}^{i, l} \sum_{k=1}^{N} R_{s S}^{i, l}(k) \gamma(k)\right] \text {, } \\
& E\left[\frac{\partial \mathfrak{L}}{\partial \kappa_{i}} \frac{\partial \mathfrak{L}}{\partial \kappa_{l}}\right]=\frac{2}{\sigma^{2}} \Re e\left[\Psi_{\text {aa }}^{i, l} \sum_{k=1}^{N} R_{s s}^{i, l}(k) \gamma(k)\right], \\
& E\left[\frac{\partial \mathfrak{L}}{\partial \tau_{i}} \frac{\partial \mathfrak{L}}{\partial \theta_{l}}\right]=\frac{2}{\sigma^{2}} \Re e\left[\Psi_{\mathbf{a a}_{\theta}^{\prime}}^{i, l} \zeta_{i}^{H} \zeta_{l} \sum_{k=1}^{N} R_{s^{\prime} s}^{i, l}(k) \gamma(k)\right], \\
& E\left[\frac{\partial \mathfrak{L}}{\partial \tau_{i}} \frac{\partial \mathfrak{L}}{\partial \phi_{l}}\right]=\frac{2}{\sigma^{2}} \Re e\left[\Psi_{\mathbf{a a}_{\phi}^{\prime}}^{i, l} \zeta_{i}^{H} \zeta_{l} \sum_{k=1}^{N} R_{s^{\prime} s}^{i, l}(k) \gamma(k)\right], \\
& E\left[\frac{\partial \mathfrak{L}}{\partial \tau_{i}} \frac{\partial \mathfrak{L}}{\partial \nu_{l}}\right]=\frac{4 \pi}{\sigma^{2}} \Im m\left[\Psi_{\text {aa }}^{i, l} \zeta_{i} \zeta_{l}^{H} \sum_{k=1}^{N} R_{s^{\prime} s}^{i, l}(k) \gamma^{H}(k) t_{k}\right], \\
& E\left[\frac{\partial \mathfrak{L}}{\partial \tau_{i}} \frac{\partial \mathfrak{L}}{\partial \eta_{l}}\right]=\frac{2}{\sigma^{2}} \Re e\left[\Psi_{\text {aa }}^{i, l} \zeta_{i}^{H} \sum_{k=1}^{N} R_{s^{\prime} s}^{i, l}(k) \gamma(k)\right], \\
& E\left[\frac{\partial \mathfrak{L}}{\partial \tau_{i}} \frac{\partial \mathfrak{L}}{\partial \kappa_{l}}\right]=\frac{-2}{\sigma^{2}} \Im m\left[\Psi_{\mathbf{a a}}^{i, l} \zeta_{i} \sum_{k=1}^{N} R_{s^{\prime} s}^{i, l}(k) \gamma^{H}(k)\right], \\
& E\left[\frac{\partial \mathfrak{L}}{\partial \theta_{i}} \frac{\partial \mathfrak{L}}{\partial \phi_{l}}\right]=\frac{2}{\sigma^{2}} \Re e\left[\zeta_{i}^{H} \zeta_{l} \Psi_{\mathbf{a}_{\theta}^{\prime} \mathbf{a}_{\phi}^{\prime}}^{i, l} \sum_{k=1}^{N} R_{s S}^{i, l}(k) \gamma(k)\right], \\
& E\left[\frac{\partial \mathfrak{L}}{\partial \theta_{i}} \frac{\partial \mathfrak{L}}{\partial \nu_{l}}\right]=\frac{4 \pi}{\sigma^{2}} \Im m\left[\Psi_{\mathbf{a a}_{\theta}^{\prime}}^{i, l} \zeta_{i} \zeta_{l}^{H} \sum_{k=1}^{N} R_{s s}^{i, l}(k) \gamma^{H}(k) t_{k}\right], \\
& E\left[\frac{\partial \mathfrak{L}}{\partial \theta_{i}} \frac{\partial \mathfrak{L}}{\partial \eta_{l}}\right]=\frac{2}{\sigma^{2}} \Re e\left[\Psi_{\mathbf{a}_{\theta}^{\prime} \mathbf{a}}^{i, l} \zeta_{i}^{H} \sum_{k=1}^{N} R_{s S}^{i, l}(k) \gamma(k)\right], \\
& E\left[\frac{\partial \mathfrak{L}}{\partial \theta_{i}} \frac{\partial \mathfrak{L}}{\partial \kappa_{l}}\right]=\frac{-2}{\sigma^{2}} \Im m\left[\Psi_{\mathbf{a}_{\theta}^{\prime} \mathbf{a}}^{i, l} \zeta_{i} \sum_{k=1}^{N} R_{s s}^{i, l}(k) \gamma^{H}(k)\right], \\
& E\left[\frac{\partial \mathfrak{L}}{\partial v_{i}} \frac{\partial \mathfrak{L}}{\partial \eta_{l}}\right]=\frac{4 \pi}{\sigma^{2}} \Im m\left[\Psi_{\mathbf{a a}}^{i, l} \zeta_{i}^{H} \sum_{k=1}^{N} R_{s S}^{i, l}(k) \gamma(k) t_{k}\right], \\
& E\left[\frac{\partial \mathfrak{L}}{\partial \nu_{i}} \frac{\partial \mathfrak{L}}{\partial \kappa_{l}}\right]=\frac{-4 \pi}{\sigma^{2}} \Re e\left[\Psi_{\mathbf{a a}}^{i, l} \zeta_{i}^{H} \sum_{k=1}^{N} R_{s S}^{i, l}(k) \gamma(k) t_{k}\right], \\
& E\left[\frac{\partial \mathfrak{L}}{\partial \eta_{i}} \frac{\partial \mathfrak{L}}{\partial \kappa_{l}}\right]=\frac{-2}{\sigma^{2}} \Im m\left[\Psi_{\text {aa }}^{i, l} \sum_{k=1}^{N} R_{s S}^{i, l}(k) \gamma^{H}(k)\right] .
\end{aligned}
$$

\section{References}

[1] M.K. Ozdemir, H. Arslan, Channel estimation for wireless OFDM systems, IEEE Commun. Surv. Tutor. 9 (2) (2007) 18-48.

[2] H. Krim, M. Viberg, Two decades of array signal processing research: the parametric approach, IEEE Signal Process. Mag. 13 (4) (1996) 67-94.

[3] J. Capon, High-resolution frequency-wavenumber spectrum analysis, Proc. IEEE 57 (8) (1969) 1408-1418.

[4] R.O. Schmidt, A signal subspace approach to multiple emitter location and spectral estimation, PhD thesis, Stanford Univ., Stanford, CA, 1981. 
[5] M. Rubsamen, A.B. Gershman, Direction-of-arrival estimation for nonuniform sensor arrays: from manifold separation to Fourier domain MUSIC methods, IEEE Trans. Signal Process. 57 (2) (2009) 588-599.

[6] E. Ozkan, M.B. Guldogan, U. Orguner, F. Gustafsson, Ground multiple target tracking with a network of acoustic sensor arrays using PHD and CPHD filters, in: IEEE Int. Conf. Information Fusion (FUSION), 2011

[7] M.R. Azimi-Sadjadi, A. Pezeshki, L. Scharf, M. Hohil, Wideband DOA estimation algorithms for multiple target detection and tracking using unattended acoustic sensors, Proc. SPIE (2004).

[8] J. Liu, Z. Huang, Y. Zhou, Extended 2Q-MUSIC algorithm for noncircular signals, Signal Process. 88 (6) (2008) 1327-1339.

[9] C.E. Kassis, J. Picheral, C. Mokbel, Advantages of nonuniform arrays using rootMUSIC, Signal Process. 90 (2) (2010) 689-695.

[10] M. Viberg, B. Ottersten, Sensor array processing based on subspace fitting, IEEE Trans. Signal Process. 39 (5) (1991) 1110-1121.

[11] M. Viberg, B. Ottersten, T. Kailath, Detection and estimation in sensor arrays using weighted subspace fitting, IEEE Trans. Signal Process. 39 (11) (1991) 2436-2449.

[12] R. Roy, T. Kailath, Esprit-estimation of signal parameters via rotational invariance techniques, IEEE Trans. Acoust., Speech, Signal Process. 37 (7) (1989) 984-995.

[13] M. Haardt, J.A. Nossek, Unitary ESPRIT how to obtain increased estimation accuracy with a reduced computational burden, IEEE Trans. Signal Process. 43 (5) (1995) 1232-1242.

14] L.C. Godara, Application of antenna arrays to mobile communications, part II: Beam-forming and direction-of-arrival considerations, Proc. IEEE 85 (8) (1997) 1195-1245.

[15] P. Stoica, A. Gershman, Maximum-likelihood DOA estimation by data-supported grid search, IEEE Signal Process. Lett. 6 (10) (1999) 273-275.

[16] I. Ziskind, M. Wax, Maximum likelihood localization of multiple sources by alternating projection, IEEE Trans. Acoust., Speech, Signal Process. 36 (10) (1988) 1553-1560.

[17] K.C. Sharman, Maximum likelihood parameter estimation by simulated annealing, in: IEEE Int. Conf. Acoust. Speech Sign. Processing (ICASSP), 1988.

[18] A.P. Dempster, N.M. Laird, D.B. Rubin, Maximum likelihood from incomplete data via the EM algorithm, J. Roy. Statist. Soc. 39 (1) (1977) 1-38.

[19] P.J. Chung, J.F. Bohme, Recursive EM SAGE-inspired algorithms with application to DOA estimation, IEEE Trans. Signal Process. 53 (8) (2005) 2664-2677.

[20] O. Cappe, M. Charbit, E. Moulines, EM Recursive, algorithm with applications to DOA estimation, in: IEEE Int. Conf. Acoust. Speech Sign. Processing (ICASSP), 2006

[21] T.Y. Al-Naffouri, An EM-based forward-backward Kalman filter for the estimation of time-variant channels in OFDM, IEEE Trans. Signal Process. 55 (7) (2007) 3924-3930.

[22] J.A. Fessler, A.O. Hero, Space-alternating generalized expectation-maximization algorithm, IEEE Trans. Signal Process. 42 (10) (1994) 2664-2677.

[23] N. Czink, X. Yin, H. Ozcelik, M. Herdin, E. Bonek, B.H. Fleury, Cluster characteristics in a MIMO indoor propagation environment, IEEE Trans. Wireless Commun. 6 (4) (2007) 1465-1475

[24] A. Kocian, E. Panayirci, H. Poor, M. Ruggieri, A Monte Carlo implementation of the SAGE algorithm for joint soft-multiuser decoding, channel parameter estimation, and code acquisition, IEEE Trans. Signal Process. 58 (11) (2010) 5756-5766.

[25] B.H. Fleury, M. Tschudin, R. Heddergott, D. Dahlhaus, K.I. Pedersen, Channe parameter estimation in mobile radio environments using the SAGE algorithm, IEEE J. Sel. Areas Commun. 17 (3) (1999) 434-450.

[26] F. Yang, J. Song, Y. Zhang, C. Pan, Z. Yang, SAGE-based estimation of doubly selective channel with an orthogonal polynomial model, Signal Process. 88 (4) (2008) 1061-1068
[27] K. Haneda, J.I. Takada, An application of SAGE algorithm for UWB propagation channel estimation, in: IEEE Int. Conf. Ultra Wideband Systems and Technologies (UWBST), 2003.

[28] P.J. Chung, J.F. Bohme, DOA estimation using fast EM and SAGE algorithms, Signal Process. 82 (2002) 1753-1762.

[29] J. Verhaevert, E.V. Lil, A.V. de Capelle, Direction of arrival (DOA) parameter estimation with the SAGE algorithm, Signal Process. 84 (2004) 619-629.

[30] N. Levanon, E. Mozeson, Radar Signals, Wiley-IEEE Press, 2004

[31] M.B. Guldogan, O. Arikan, Multipath channel identification by using global optimization in ambiguity function domain, Signal Process. 91 (11) (2011) 26472660.

[32] A.J. Paulraj, C.B. Papadias, Space-time processing for wireless communications, IEEE Signal Process. Mag. 14 (6) (1997) 49-83

[33] T.S. Rappaport, Wireless Communications Principles and Practice, Prentice Hall, 2002.

[34] M. Wax, T. Kailath, Detection of signals by information theoretic criteria, IEEE Trans. Acoust., Speech, Signal Process. 33 (2) (1985) 387-392.

[35] R.F. Brcich, A.M. Zoubir, P. Pelin, Detection of sources using bootstrap techniques, IEEE Trans. Signal Process. 50 (2) (2002) 206-215.

[36] P.J. Chung, J.F. Bohme, C.F. Mecklenbrauker, A.O. Hero, Detection of the number of signals using the Benjamini-Hochberg procedure, IEEE Trans. Signal Process. 55 (6) (2007) 2497-2508.

[37] J. Liang, Joint estimation of source number and DOA using simulated annealing algorithm, Digital Signal Process. 20 (3) (2010) 887-899.

[38] A. Goldsmith, Wireless Communications, Cambridge University Press, 2005

[39] M.B. Guldogan, O. Arikan, Comparison of the CAF-DF and SAGE algorithms in multipath channel parameter estimation, in: IEEE Wksp. Sensor Array and Multichannel Signal Processing (SAM), 2008.

[40] P.M. Woodward, Probability and Information Theory with Application to Radar, Pergamon, London, 1953.

[41] B.R. Mahafza, MATLAB Simulations for Radar Systems Design, Chapman and Hall/CRC, 2003.

[42] P.Z. Peebles, Radar Principles, Wiley, 1998.

[43] S. Kay, Fundamentals of Statistical Signal Processing: Estimation Theory, Prentice Hall, 1993.

[44] P. Stoica, A. Nehorai, MUSIC, maximum likelihood, and Cramer-Rao bound, IEEE Trans. Acoust., Speech, Signal Process. 37 (5) (1989) 720-741.

Mehmet Burak Guldogan received the B.S., M.S. and Ph.D. degrees all in Electrical and Electronics Engineering from the Bilkent University, Turkey, in 2003, 2006 and 2010, respectively. Since 2010, he has been a postdoctoral fellow in the Automatic Control Group at the Linköping University, Sweden. His current research interests are in statistical signal processing, time-frequency analysis, array signal processing and target tracking.

Orhan Arikan received the B.Sc. degree in Electrical and Electronics Engineering from the Middle East Technical University in 1986 and both the M.S. and Ph.D. degrees in Electrical and Computer Engineering from the University of Illinois, Urbana-Champaign, in 1988 and 1990, respectively. Following his graduate studies, he worked for three years as a Research Scientist at the Schlumberger-Doll Research, Ridgefield, CT. He joined Bilkent University in 1993, where he is presently Professor of Electrical Engineering since 2006. His current research interests are in statistical signal processing, time-frequency analysis and array signal processing. 